\title{
BRIDGES FOR PEDESTRIANS WITH RANDOM PARAMETERS USING THE STOCHASTIC FINITE ELEMENTS ANALYSIS
}

\author{
J. SZAFRAN and M. KAMIŃSKI* \\ Department of Structural Mechanics, Faculty of Civil Engineering \\ Architecture and Environmental Engineering \\ Technical University of Łódź \\ Al. Politechniki 6, 90-924 Łódź, POLAND \\ E-mail: Jacek.Szafran@p.lodz.pl; Marcin.Kaminski@p.lodz.pl
}

\begin{abstract}
The main aim of this paper is to present a Stochastic Finite Element Method analysis with reference to principal design parameters of bridges for pedestrians: eigenfrequency and deflection of bridge span. They are considered with respect to random thickness of plates in boxed-section bridge platform, Young modulus of structural steel and static load resulting from crowd of pedestrians. The influence of the quality of the numerical model in the context of traditional FEM is shown also on the example of a simple steel shield. Steel structures with random parameters are discretized in exactly the same way as for the needs of traditional Finite Element Method. Its probabilistic version is provided thanks to the Response Function Method, where several numerical tests with random parameter values varying around its mean value enable the determination of the structural response and, thanks to the Least Squares Method, its final probabilistic moments.
\end{abstract}

Key words: bridges for pedestrians, eigenfrequencies, Stochastic Finite Element Method, Response Function Method, generalized stochastic perturbation technique, reliability analysis.

\section{Introduction}

One of the main requirements referring to bridges for pedestrians $[1,2]$, next to ones related to security of the users (capacity), is a comfort of exploitation. A very special group of these requirements is a group called vibrational (dynamical) comfort $[3,4]$. It contains a set of parameters, which make it possible to keep a structure in a dynamical regime that will not cause a physical and mental discomfort in the users. We can distinguish excessive vibrations and deflections of the bridge platform in this group. According to the British Standard BS 5400, for instance, the maximum admissible vertical acceleration when passing one person should be less than

$$
a_{1} \leq 0.5 \sqrt{f_{1}}
$$

where $f_{l}$ is the fundamental eigenfrequency of the transverse vibrations. Acceleration induced by a passage of one person is described in the design process by the following formula

$$
a_{1}=4 \pi^{2} f_{1}^{2} y K \psi
$$

where: $y$ means a deflection of the platform resulting from the concentrated load equal to $0.7 \mathrm{kN}, \mathrm{K}$ is a configuration factor, whose value depends on the number of the spans, $\psi$ stands for the dynamic response factor dependent on the type of the platform. The basic parameter describing acceleration caused by a single

\footnotetext{
* To whom correspondence should be addressed
} 
person in the formula above as one can see is the fundamental frequency of eigenvibration, particularly when this frequency is in a range of frequency of the human steps $\left(1.5<f_{1}<2.4 \mathrm{~Hz}\right)$; it is very disadvantageous because of a possibility of excitation of the structure by the pedestrian crowd [5]. Another important factor is the vertical displacement of the steel bridge structure, caused by static load. The maximum vertical displacement obtained in calculations and design process must be limited not to cause discomfort for users. Limitations are given in design codes and they take different values, for example $y<l / 380$ (Polish code) and $y<l / 200$ (British code) where $l$ is a span length.

Basic assumptions of the structural reliability are provided by partial safety factors in current design codes requirements. They are defined by sophisticated probabilistic methods [6] and also by the advanced research approaches. A structural analysis accounting for the parameters uncertainty enables computational modeling of the problems in designing, optimization and, first of all, reliability assessment of the civil engineering structures. An uncertainty of the parameters, decisive for the structure's effort, may have quite different sources, for instance, structural defects, material degradation, variability in time, manufacturing and erecting inaccuracies as well as geometrical imperfections and physical parameters.

One of the approaches mentioned above is proposed here and belongs to the family of perturbation methods - it is based on a given order polynomial representation of the desired output parameter with respect to the given random input. Numerical recovery of this function (or these functions in its local version) is provided through the series of classical FEM experiments and the Least Squares Method (LSM) carried out in the desired FEM system, while probabilistic moments and coefficients are calculated in the computer algebra system using the perturbation-based equations (MAPLE, v. 13). It is necessary to underline that a derivation of the specific analytical formulas for those output random quantities is an inherent part of the stochastic perturbation method, quite contrary to the simulation technique, where statistical estimators have quite general character, independent of the problem being solved. From the numerical point of view we have performed the shear test of a steel shield in plane strain analysis to present the proposed method in its extended version. However, the main objective of this paper is a presentation of an influence of random parameters on two very important factors: vertical displacement and eigenfrequencies of the bridge platform. Analyses of those factors were performed with respect to a few random input parameters such as Young modulus, thickness of the plates in box platform, static load of the pedestrian crowd.

\section{Governing equations}

Consider the following linear elasto-dynamic problem $[7,8,9]$ consisting of:

- the equations of motion

$$
D^{T} \boldsymbol{\sigma}+\hat{\boldsymbol{f}}=\rho \ddot{\boldsymbol{u}}, \quad \boldsymbol{x} \in \Omega, \quad \tau \in\left[t_{0}, \infty\right),
$$

- the constitutive equations

$$
\boldsymbol{\sigma}=\boldsymbol{C} \boldsymbol{\varepsilon}, \quad \boldsymbol{x} \in \Omega, \quad \tau \in\left[t_{0}, \infty\right),
$$

- $\quad$ the geometric equations

$$
\varepsilon=D \boldsymbol{u}, \quad \boldsymbol{x} \in \Omega, \quad \tau \in\left[t_{0}, \infty\right),
$$

- the displacement boundary conditions

$$
\boldsymbol{u}=\hat{\boldsymbol{u}}, \quad \boldsymbol{x} \in \partial \Omega_{u}, \quad \tau \in\left[t_{0}, \infty\right),
$$


- the stress boundary conditions

$$
\boldsymbol{n} \boldsymbol{\sigma}=\hat{\boldsymbol{t}}, \quad \boldsymbol{x} \in \partial \Omega_{\sigma}, \quad \tau \in\left[t_{0}, \infty\right),
$$

- the initial conditions

$$
\boldsymbol{u}=\hat{\boldsymbol{u}}^{0}, \quad \dot{\boldsymbol{u}}=\hat{\dot{\boldsymbol{u}}}^{\mathbf{0}}, \quad \tau=t_{0} .
$$

Assume that all the state functions appearing in this system are sufficiently smooth functions of the independent variables $\boldsymbol{x}$ and $\tau$. Let us consider the variation $\boldsymbol{u}(\boldsymbol{x}, \tau)$ at certain time moment $\tau=t$ denoted by $\delta \boldsymbol{u}(\boldsymbol{x}, \tau)$. Using the above equations one can show that

$$
-\int_{\Omega}\left(D^{T} \sigma+\hat{f}-\rho \ddot{u}\right)^{T} \delta u d \Omega+\int_{\partial \Omega_{\sigma}}(n \sigma-\hat{t})^{T} \delta u d(\partial \Omega)=0 .
$$

Assume further that the displacement function $\boldsymbol{u}(\boldsymbol{x}, t)$ has known values at the initial moment $\boldsymbol{u}\left(\boldsymbol{x}, t_{1}\right)=0$ and at the final moment $\boldsymbol{u}\left(\boldsymbol{x}, t_{2}\right)=0$, so that the variations of this function also equal 0 at these time moments, which means $\delta \boldsymbol{u}\left(\boldsymbol{x}, t_{1}\right)=0, \delta \boldsymbol{u}\left(\boldsymbol{x}, t_{2}\right)=0$. Integrating by parts one can obtain that

$$
\int_{\boldsymbol{t}_{1}}^{\boldsymbol{t}_{2}}\left[\delta \boldsymbol{T}-\int_{\boldsymbol{\Omega}} \boldsymbol{\sigma}^{\boldsymbol{T}} \delta \boldsymbol{\varepsilon} d \boldsymbol{\Omega}+\int_{\boldsymbol{\Omega}} \hat{\boldsymbol{f}}^{\boldsymbol{T}} \delta \boldsymbol{u} d \boldsymbol{\Omega}+\int_{\partial \boldsymbol{\Omega}} \hat{\boldsymbol{t}}^{\boldsymbol{T}} \delta \boldsymbol{u} d(\partial \boldsymbol{\Omega})\right] d \tau=0
$$

where the kinetic energy of the region $\Omega$ is defined as

$$
T=\frac{1}{2} \int_{\Omega} \rho \dot{\boldsymbol{u}}^{T} \dot{\boldsymbol{u}} d \Omega
$$

Notice also that

$$
\delta \boldsymbol{\varepsilon}=\boldsymbol{D} \delta \boldsymbol{u}, \quad \boldsymbol{x} \in \Omega, \quad \tau \in\left[t_{0}, \infty\right) .
$$

Next, it is assumed that the mass forces $\hat{\boldsymbol{f}}$ and the surface loadings $\hat{\boldsymbol{t}}$ are independent from the displacement vector $u$ and, therefore, Eq.(2.8) can be modified to the following statement:

$$
\delta \int_{\boldsymbol{t}_{1}}^{\boldsymbol{t}_{2}}\left(T-J_{p}\right) d \tau=0
$$

where $\boldsymbol{J}_{p}$ denotes potential energy stored in the entire domain $\Omega$

$$
J_{p}=U-\int_{\boldsymbol{\Omega}} \hat{\boldsymbol{f}}^{T} \boldsymbol{u} d \Omega-\int_{\partial \mathbf{\Omega}_{\boldsymbol{\sigma}}} \hat{\boldsymbol{t}}^{T} \boldsymbol{u} d(\partial \Omega)=0
$$


whereas $U$ is the elastic strain energy given by the formula

$$
U=\frac{1}{2} \int_{\Omega} \varepsilon^{T} C \varepsilon d \Omega
$$

It is well known that Eq.(2.11) represents the Hamilton principle widely employed in structural dynamics [10] as the basis for further Finite Element Method and its stochastic counterpart implementations.

\section{Probabilistic perturbation technique description}

Let us introduce next the random variable $b \equiv b(\omega)$ with its probability density function as $p(b)$. The $m^{\text {th }}$ central probabilistic moment is defined as

$$
\mu_{m}(b)=\int_{-\infty}^{+\infty}(b-E[b])^{m} p_{b}(x) d x
$$

The basic idea of the stochastic perturbation approach $[11,12]$ is to expand all the input variables and the state functions via the Taylor series about their spatial expectations using some small parameter $\varepsilon>0$. In case of some random quantity $e=e(b)$, the following expression is employed

$$
e=e^{0}+\sum_{n=1}^{\infty} \frac{1}{n !} \varepsilon^{n} \frac{\partial^{n} e}{\partial b^{n}}(\Delta b)^{n}
$$

where $\varepsilon \Delta b=\varepsilon\left(b-b^{0}\right)$ is the first variation of $b$ about $b^{0}$, where the symbol $(.)^{0}$ represents the function value (.) taken at the expectation $b^{0}$. Let us analyze further the expected values of any state function $f(b)$ defined according to Eq.(3.2) by its expansion via the Taylor series with a given small parameter $\varepsilon$ (taken as equal to 1 in numerous practical computations) as follows

$$
E[f(b)]=\int_{-\infty}^{+\infty} f(b) p(b) d b=\int_{-\infty}^{+\infty}\left(f^{0}(b)+\sum_{n=1}^{\infty} \frac{1}{n !} \varepsilon^{n} \frac{\partial^{n} f}{\partial b^{n}} \Delta b^{n}\right) p(b) d b .
$$

From the numerical point of view, the expansion introduced by Eq.(3.2) is carried out for the summation over the finite number of components and with finite limits during the integration process. Now, let us focus on an analytical derivation of the probabilistic moments for the structural response function. It is easy to prove that the general $8^{\text {th }}$ order expansion results in the formula

$$
E[f(b)]=\left.f^{0}(b)\right|_{b=b^{0}}+\left.\frac{1}{2} \varepsilon^{2} \mu_{2}(b) \frac{\partial^{2} f}{\partial b^{2}}\right|_{b=b^{0}}+\ldots+\left.\frac{1}{8 !} \varepsilon^{8} \mu_{8}(b) \frac{\partial^{8} f}{\partial b^{8}}\right|_{b=b^{0}}
$$

where the even components in case of Gaussian variables are dropped off. Thanks to such an extension of the random output, any desired efficiency of the expected values as well as higher probabilistic moments can be achieved by an appropriate choice of the distribution parameters.

Finally, one may recover the kurtosis, skewness and coefficient of variance $[13,14]$ as 


$$
\kappa(f(b))=\frac{\mu_{4}(f(b))}{\sigma^{4}(f(b))}-3, \quad \beta(f(b))=\frac{\mu_{3}(f(b))}{\sigma^{3}(f(b))}, \quad \alpha(f(b))=\frac{\sigma(f(b))}{E[f(b)]} .
$$

The remaining formulas derived in the same manner (for Gaussian random variables) describing the following probabilistic moments: variance (in the expansion of $16^{\text {th }}$ order), third central moment $\left(18^{\text {th }}\right.$ order) and fourth central moment ( $20^{\text {th }}$ order) are given in the Appendix.

The expected value of fundamental eigenfrequencies of the bridge for pedestrians $f_{l}$ with respect to random thickness of the plates in the box formed steel platform $t$ is described in numerical experiment given below, as follows

$$
E\left[f_{l}(t)\right]=\left.f^{0}\left(f_{l}(t)\right)\right|_{t=t^{0}}+\left.\frac{1}{2} \varepsilon^{2} \mu_{2}(t) \frac{\partial^{2} f\left(f_{l}(t)\right)}{\partial t^{2}}\right|_{t=t^{0}}+\ldots+\left.\frac{1}{8 !} \varepsilon^{8} \mu_{8}(t) \frac{\partial^{8} f\left(f_{l}(t)\right)}{\partial t^{8}}\right|_{t=t^{0}}
$$

The function $f\left(f_{l}(t)\right)$ can be approximated with using the Least Square Method for $\mathrm{m}^{\text {th }}$ degree polynomials (the degree of polynomial depends on the required order of perturbation technique)

$$
f\left(f_{1}(t)\right)=a_{0}+a_{1} t+a_{2} t^{2}+\ldots+a_{m} t^{m}
$$

The required given set of data $\left\{\left(t_{1} ; f_{l}\left(t_{1}\right)\right),\left(t_{2} ; f_{l}\left(t_{2}\right)\right), \ldots\left(t_{n} ; f_{l}\left(t_{n}\right)\right)\right\}$ where $n \geq m+1$ the best fitting curve $f\left(f_{l}(t)\right)$ exhibits the least squares error, i.e.,

$$
\Pi=\min \sum_{i=1}^{n}\left[f_{l}\left(t_{i}\right)-f\left(f_{l}\left(t_{i}\right)\right)\right]^{2}=\min \sum_{i=1}^{n}\left[f_{l}\left(t_{i}\right)-\left(a_{0}+a_{1} t_{i}+a_{2} t_{i}^{2}+\ldots+a_{m} t_{i}^{m}\right)\right]^{2} .
$$

Minimization procedures are implemented with the use of the gradient method, where all partial derivatives are calculated with respect to all the coefficients $a_{i}$ separately. The entire computational procedure has been implemented into the computer algebra system MAPLE v.13, where all probabilistic moments are also derived and determined.

Probabilistic moments of the Gaussian distribution appearing in the above equations are equal to

$$
\mu_{R}(b)=\left\{\begin{array}{cc}
0, & k=2 j+1 \\
\sigma^{(2 j)}(b) \frac{2^{j}+\left(\frac{2 j+1}{2}\right)}{\sqrt{\pi}}, & k=2 j
\end{array}\right.
$$

\section{Computational implementation}

Let us consider the following discretization of the displacement field $\boldsymbol{u}(\boldsymbol{x}, \tau)$

$$
u^{\alpha}(x, \tau) \cong \phi(x) q^{\alpha}(\tau), \quad u^{\alpha}(x, \tau) \cong \Phi(x) r^{\alpha}(\tau)
$$

where $\boldsymbol{q}$ is a vector of the generalized coordinates for the finite element considered, $\boldsymbol{r}$ is a vector for the generalized coordinates of the entire discretized system. The generalized coordinates vector for the entire structure model is composed of the finite element degrees of freedom and the transformation matrix as 


$$
\boldsymbol{r}^{\alpha}=\boldsymbol{a q ^ { \alpha }}
$$

$\boldsymbol{\varphi}$ and $\boldsymbol{\Phi}$ are the corresponding shape function matrices (local and global). Contrary to the classical formulations of both FEM and the perturbation-based Stochastic Finite Element Method, we introduce here an additional index $\alpha=1, \ldots, M$ to distinguish between various solutions of the elasto-dynamic problem necessary to recover the response functions. The strain tensor can be expressed as

$$
\boldsymbol{\varepsilon}^{\alpha}(\boldsymbol{x}, \tau)=\boldsymbol{B}(\boldsymbol{x}) \boldsymbol{q}^{\alpha}(\tau)=\tilde{B}(\boldsymbol{x}) \boldsymbol{r}^{\alpha}(\tau)
$$

The discretized version of Hamilton's principle is obtained as

$$
\delta \int_{t_{1}}^{t_{2}}\left(\frac{1}{2} \sum \boldsymbol{q}^{\alpha T} \boldsymbol{m}^{\alpha} \boldsymbol{q}^{\alpha}-\frac{1}{2} \sum \boldsymbol{q}^{\alpha T} \boldsymbol{k}^{\alpha} \boldsymbol{q}^{\alpha}+\sum \boldsymbol{Q}^{\alpha T} \boldsymbol{q}^{\alpha}\right) d \tau=0
$$

where a summation is made along with all finite elements in the system, so that we have in a global description

$$
\delta \int_{\boldsymbol{t}_{1}}^{\boldsymbol{t}_{2}}\left(\frac{1}{2} \dot{\boldsymbol{r}}^{\alpha T} \boldsymbol{M}^{\alpha} \dot{\boldsymbol{r}}^{\alpha}-\frac{1}{2} \boldsymbol{r}^{\alpha T} \boldsymbol{K}^{\alpha} \boldsymbol{r}^{\alpha}+\boldsymbol{R}^{\alpha T} \boldsymbol{r}^{\alpha}\right) d \tau=0
$$

and where the global mass matrix and stiffness matrix are defined as

$$
\boldsymbol{M}^{\alpha}=\int_{\Omega} \rho^{\alpha}(\boldsymbol{x}) \tilde{\boldsymbol{B}}^{T}(\boldsymbol{x}) \tilde{\boldsymbol{B}}(\boldsymbol{x}) d \Omega, \quad \boldsymbol{K}^{\alpha}=\int_{\Omega} \tilde{\boldsymbol{B}}^{T} \boldsymbol{C}^{\alpha} \tilde{\boldsymbol{B}} d \Omega
$$

and since the 3D bar and beam elements are used in further computations (linearly depending on the Young modulus), only the first partial derivatives differ from 0. Hence, Eq.(4.5) can be rewritten as

$$
\dot{\boldsymbol{r}}^{\alpha \boldsymbol{T}} \boldsymbol{M}^{\alpha} \delta \boldsymbol{r}-\int_{t_{l}}^{t_{2}}\left(\ddot{\boldsymbol{r}}^{\alpha \boldsymbol{T}} \boldsymbol{M}^{\alpha}+\boldsymbol{r}^{\alpha \boldsymbol{T}} \boldsymbol{K}^{\alpha}-\boldsymbol{R}^{\alpha \boldsymbol{T}}\right) \delta \boldsymbol{r} d \tau=0 .
$$

We finally obtain the dynamic equilibrium system under the assumptions that $\delta \boldsymbol{r}\left(t_{1}\right)=0, \delta \boldsymbol{r}\left(t_{2}\right)=0$

$$
\boldsymbol{M}^{\alpha} \ddot{\boldsymbol{r}}^{\alpha}+\boldsymbol{K}^{\alpha} \boldsymbol{r}^{\alpha}=\boldsymbol{R}^{\alpha}
$$

which represents the equations of motion of the discretized system. Once we complete this equation with the component $\boldsymbol{C}^{\alpha} \boldsymbol{r}^{\alpha}$ by getting

$$
\boldsymbol{M}^{\alpha} \ddot{\boldsymbol{r}}^{\alpha}+\boldsymbol{C}^{\varepsilon} \dot{\boldsymbol{r}}^{\alpha}+\boldsymbol{K}^{\alpha} \boldsymbol{r}^{\alpha}=\boldsymbol{R}^{\alpha}
$$

then we decompose the damping matrix as $\boldsymbol{C}^{\alpha}=\alpha_{0} \boldsymbol{M}^{\alpha}+\alpha_{1} \boldsymbol{K}^{\alpha}$, so that

$$
\boldsymbol{M}^{\alpha} \ddot{\boldsymbol{r}}^{\alpha}+\alpha_{0} \boldsymbol{M}^{\alpha} \dot{\boldsymbol{r}}^{\alpha}+\alpha_{1} \boldsymbol{K}^{\alpha} \dot{\boldsymbol{r}}^{\alpha}+\boldsymbol{K}^{\alpha} \boldsymbol{r}^{\alpha}=\boldsymbol{R}^{\alpha}
$$

here no summation over the doubled indices $\alpha$ is applied here. As it is known, the case of the undamped free vibrations leads to the following algebraic system: 


$$
\boldsymbol{M}^{\alpha} \ddot{\boldsymbol{r}}^{\alpha}+\boldsymbol{K}^{\alpha} \boldsymbol{r}^{\alpha}=\mathbf{0}
$$

and the solution $\boldsymbol{r}^{\alpha}=\boldsymbol{A}^{\alpha} \sin \omega_{\alpha} t$ leads to the relation

$$
-\boldsymbol{M}^{\alpha} \boldsymbol{A}^{\alpha} \omega_{\alpha}^{2} \sin \omega_{\alpha} t+\boldsymbol{K}^{\alpha} \boldsymbol{A}^{\alpha} \sin \omega_{\alpha} t=\boldsymbol{0},
$$

so that for $\sin \omega_{\alpha} t \neq \boldsymbol{0}$ and $\boldsymbol{A}^{\alpha} \neq \boldsymbol{0}$ there holds

$$
-\boldsymbol{M}^{\alpha} \omega_{\alpha}^{2}+\boldsymbol{K}^{\alpha}=\mathbf{0}
$$

Some alternative methods for the determination of the eigenvalues of engineering systems with random parameters can be found in [15]. Obviously, Eq.(4.8) for the time independent of generalized coordinates reflects the well-known linear statics equilibrium systems for the RFM as follows

$$
\boldsymbol{K}^{\alpha} \boldsymbol{r}^{\alpha}=\boldsymbol{R}^{\alpha}
$$

In the next section, which fully concerns numerical experiments, we use different types of the finite elements especially in the main example - the bridge for pedestrians. The second equation (4.6) defines the general formula for stiffness matrix. It is worth recalling the formulas describing stiffness matrices for typical finite elements used in further computations:

- two-noded 2D truss linear element

$$
\boldsymbol{k}=\frac{E A}{l}\left[\begin{array}{ll}
1 & 0 \\
0 & 1
\end{array}\right]
$$

- $\quad$ two-noded 2D beam linear element

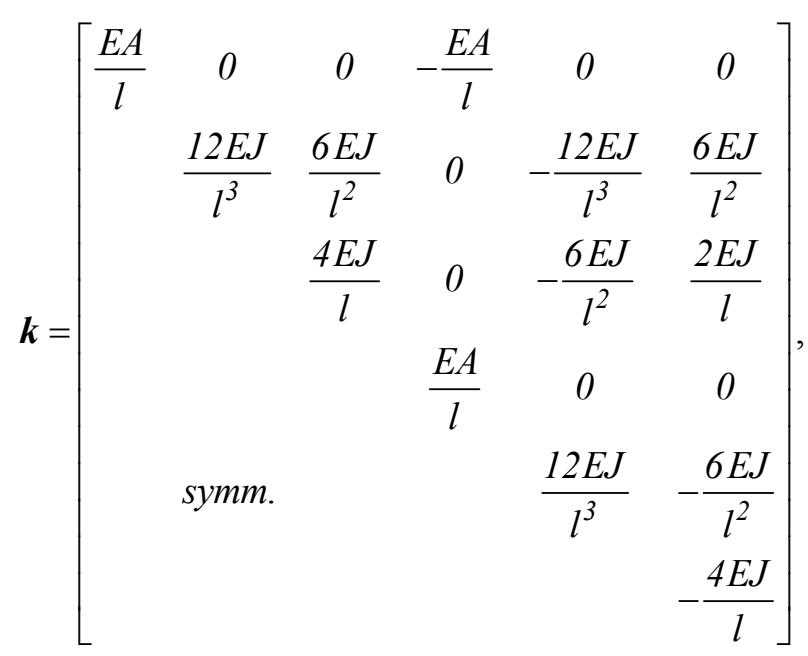

- four-noded quadrilateral shell element

$$
k=B^{T} C B
$$


where $\boldsymbol{B}$ denotes the matrix of shape functions derivatives classical for the FEM, while $\boldsymbol{C}$ is the elasticity tensor defined for the plate element as

$$
\boldsymbol{C}=\frac{E t_{p}{ }^{3}}{12\left(1-v^{2}\right)}\left[\begin{array}{ccc}
1 & v & 0 \\
& 1 & 0 \\
\text { symm. } & & \frac{1-v}{2}
\end{array}\right] .
$$

All the afore mentioned formulas show that a degree of polynomial being the response function may be initially predicted from the stiffness matrix form.

\section{Computational experiments}

\subsection{Probabilistic convergence of the Stochastic Finite Element Method}

The first computational example presents the Generalized Stochastic Perturbation Method and approach of higher orders accuracy and also contains a comparison of probabilistic moments with respect to mesh density of a structure. We analyze a steel shield given schematically in Fig. 1 with general dimensions $5.0 \mathrm{~m} \times 1.0 \mathrm{~m}$, where the left vertical edge is fully restrained and the right one is loaded with uniformly distributed tangent load. An analysis was carried out for the shear test with the Young modulus of structural steel given here as a random parameter with an expected value $E[e]$ equal to $210 \mathrm{GPa}$ (Poisson's ratio $v=0.3$ ) and Gaussian distribution. We have used three essentially different quality meshes to build a model of the shield - 20 (coarse mesh), 100 (moderate mesh) and 1600 (fine mesh) 4-noded rectangular plane strain elements.

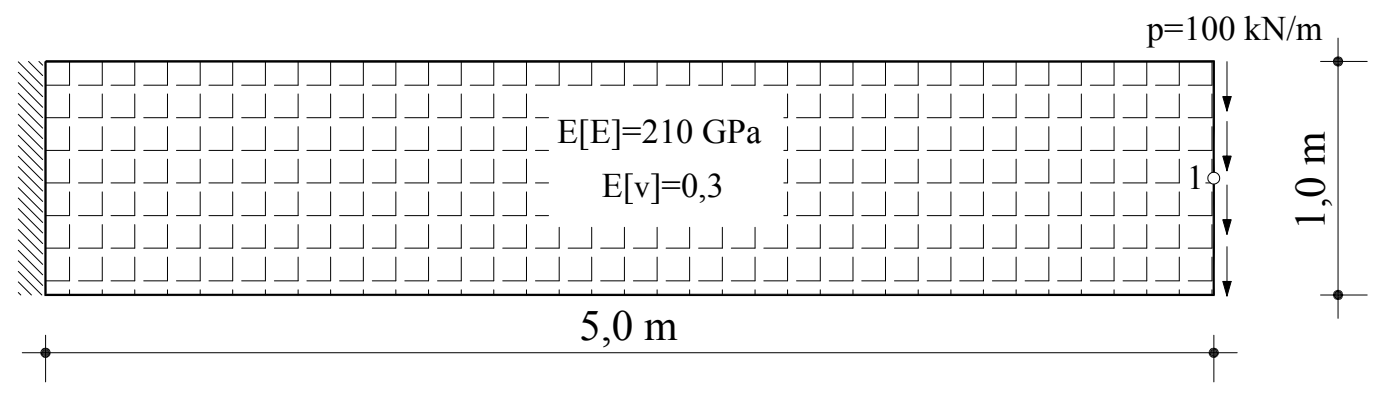

Fig.1. Static scheme of a shield.

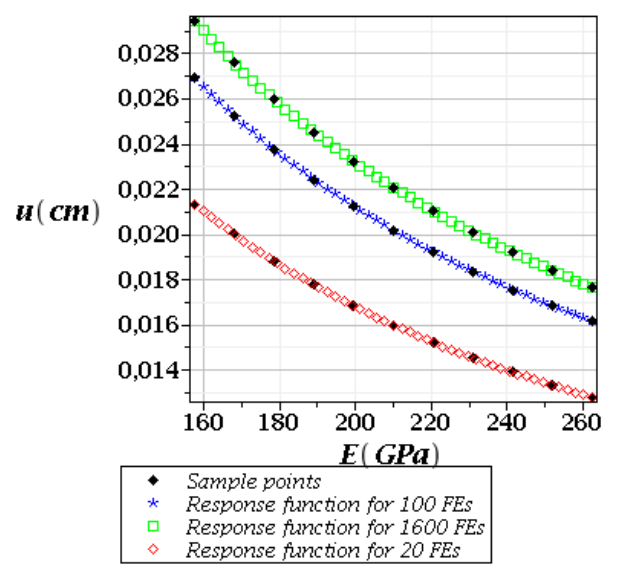

Fig.2. Mesh sensitivity of the response function of the maximum vertical deflection of a shield. 
For each model we have conducted 11 computations with different values of the Young modulus and as an outcome we have obtained a vertical displacement in point 1 (marked in Fig.1). The main objective of this test is just to check whether probabilistic moments in SFEM behave like their deterministic counterparts in traditional FEM tending all to their real values by increasing the initial mesh. All the computational analyses were performed using the commercial FEM engineering package Autodesk Robot Structural Analysis 2012 supported in probabilistic calculus with symbolic analysis system MAPLE, v.13. The nonlinear response functions for all meshes are presented in Fig. 2 and concern the vertical displacement of point 1. As we can observe all functions fit perfectly the corresponding sets of trial points without any local oscillations, disorders etc.
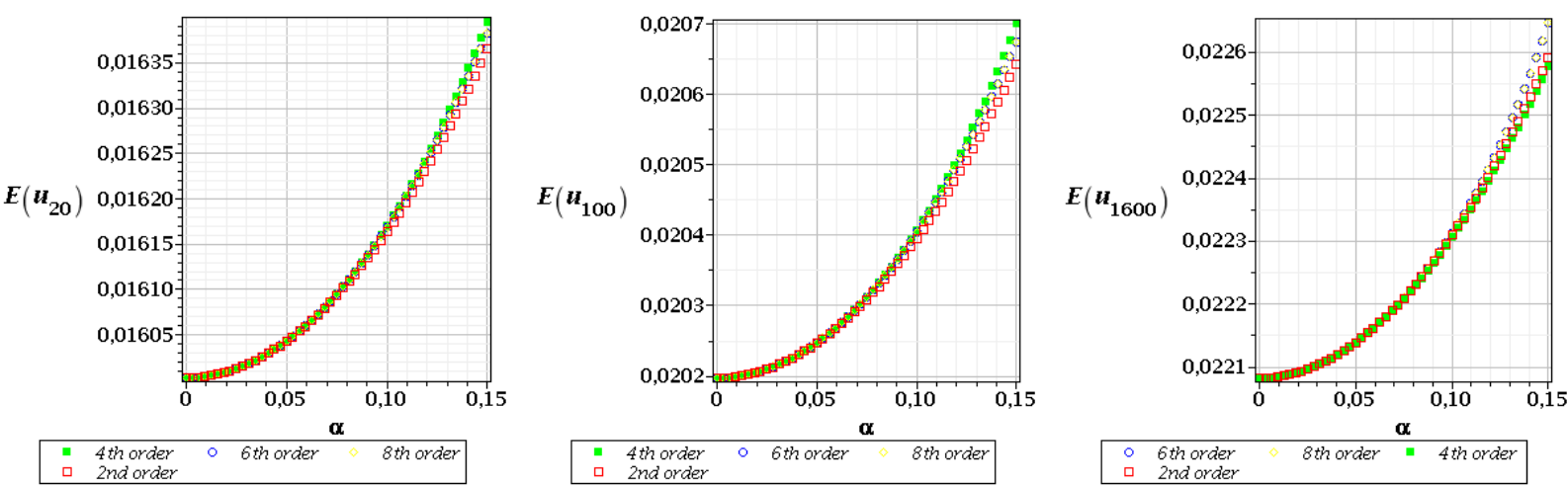

Fig.3. Expected values of the maximum vertical deflection for a coarse (left), moderate (middle) and fine (right) mesh of a shield.

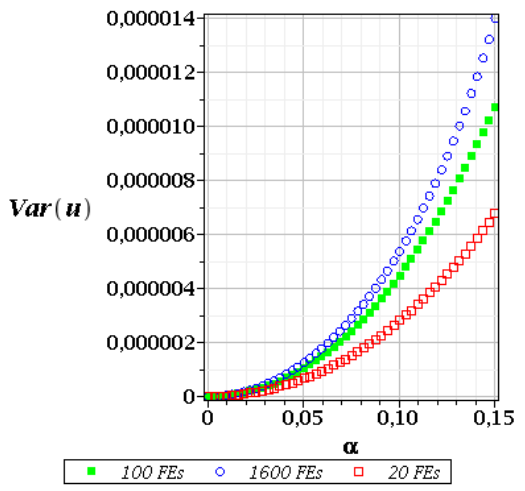

Fig.4. Comparison of variances of the maximum vertical deflection obtained for the $16^{\text {th }}$ order of perturbation approach and different response functions.

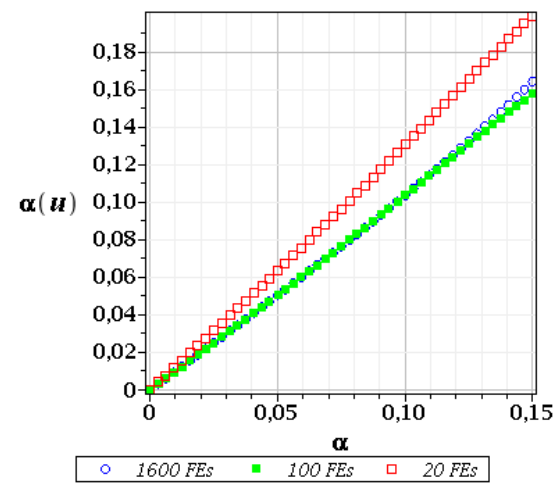

Fig.5. Comparison of coefficients of variation of the maximum vertical deflection obtained for the $8^{\text {th }}$ order of perturbation approach and different response functions. 

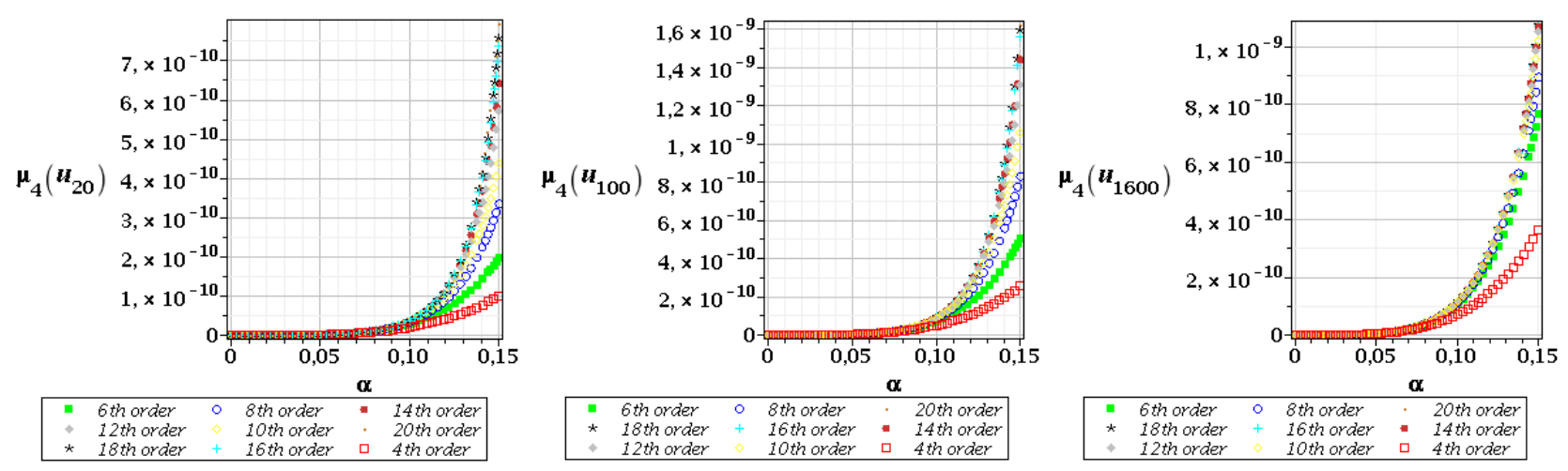

Fig.6. Fourth central moments of the maximum vertical deflection for a coarse (left), moderate (middle) and fine (right) mesh of a shield.
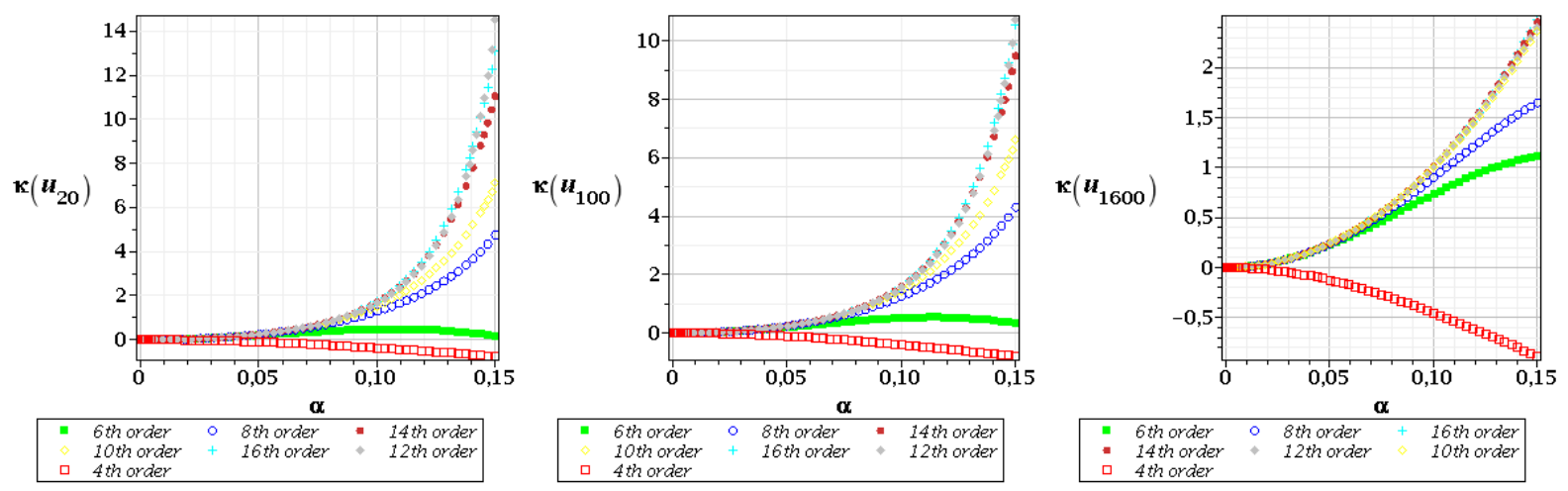

Fig.7. Kurtosis of the maximum vertical deflections for a coarse (left), moderate (middle) and fine (right) mesh of a shield.
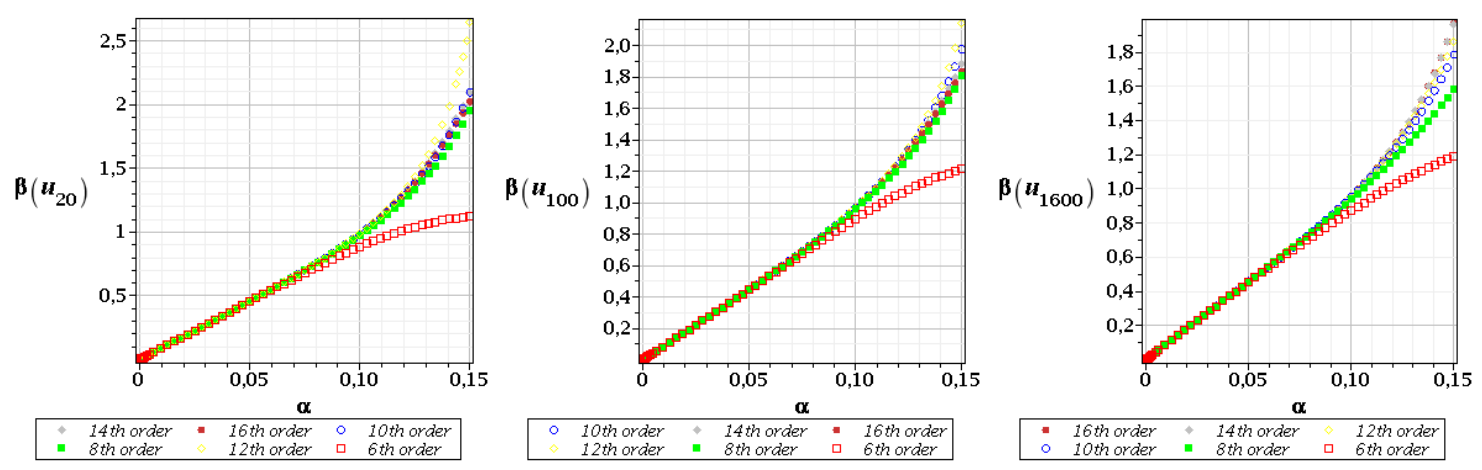

Fig.8. Skewness of the maximum vertical deflections for a coarse (left), moderate (middle) and fine (right) mesh of a shield.

Probabilistic output for the structural response is given as the expectations (Fig.3), variances (Fig.4) and coefficients of variation (Fig.5 ) for various meshes and also fourth central moments (Fig.6) kurtosis (Fig.7) and skewness (Fig.8); all these parameters are plotted with respect to the input coefficient of variation ranging from 0.0 up to 0.15 .

The expected values of vertical displacement shown in Fig. 3 reflect the influence of input random dispersion and their values increase moderately together with coefficient $\alpha$. What can also be found is that 
the mesh density strongly affects the total values of observed probabilistic moments, and this conclusion applies to all the three diagrams with expectations. In accordance to the previous numerical experiments the curves corresponding to the different orders $\left(2^{\text {nd }}, 4^{\text {th }}, 6^{\text {th }}, 8^{\text {th }}\right)$ all stabilize together systematically. The variances obtained for the $16^{\text {th }}$ order of perturbation method are visualized in Fig.4 and they show the differences with respect to the mesh density - of course the values of variance are small but also the computational example is quite ordinary but this effect may be more significant for complex systems. Input and output coefficients of variation demonstrate a linear convergence and we can also observe that the coarse model can lead us to inaccurate conclusions. In this case the results obtained for the moderate and fine meshes give us almost the same results. Functions describing the fourth probabilistic central moment in this analysis are expanded up to the twentieth perturbation order (Fig.6) as they are detected before as demanding the largest Taylor expansions [16] for accurate computations. The values of this probabilistic parameter are increasing with both - the quality of the model and the input coefficient $\alpha$. A development of higher perturbations orders gives us expected and accurate probabilistic convergence, especially in a combination with an adequate numerical model of this structure. The most spectacular differences of the values with respect to the numerical model are shown in Figs 7 and 8 - the diagrams of kurtosis and skewness, respectively. These coefficients related to the vertical displacement in a model with a fine mesh take values dramatically lower than for coarse and moderate meshes. We need to underline that there is a need to expand the response function up to the $16^{\text {th }}$ order to obtain sufficient convergence of the perturbation method. This conclusion is very important especially for more complex numerical experiments. It will be specifically proven in the next computational experiment. We can say also with no doubt that a final distribution of vertical displacement is non-Gaussian, which strongly affects the final form of the reliability index for this case study.

\subsection{Stochastic analysis of the pedestrian bridge}

The second numerical example entirely concerns the steel-concrete small bridge for pedestrians. The bridge is a steel arch structure with a suspended span platform with theoretical length equal to $48.0 \mathrm{~m}$. Principal geometrical dimensions are given in Figs 9 and 10 and are also collected in Tab.1.

Table 1. Geometrical parameters characteristics of the analyzed footbridge.

\begin{tabular}{|l|l|}
\hline Theoretical span length & $48,00 \mathrm{~m}$ \\
\hline Height of the arcs & $14,87 \mathrm{~m}$ \\
\hline Total width & $7,79 \mathrm{~m}$ \\
\hline Platform width & $3,59 \mathrm{~m}$ \\
\hline Platform height & $0,76 \mathrm{~m}$ \\
\hline Usable width & $3,50 \mathrm{~m}$ \\
\hline Materials \\
\hline Platform & Steel \\
\hline Arcs & Steel \\
\hline Lateral ramps & Reinforced concrete \\
\hline
\end{tabular}

Two high arches made using circular tubes $(508 \mathrm{~mm} \times 16 \mathrm{~mm}$ - diameter versus thickness) are inclined inside in a way that they intersect each other at $1 / 4$ of the span length. The tubes are linked by two tubular elements $(32.9 \mathrm{~mm} \times 14 \mathrm{~mm})$ in the middle section. Arches are fixed to rectangular boxed bars with a cross section equal to $750 \mathrm{~mm} \times 1000 \mathrm{~mm}$. The platform has a box cross section with seven cells and $0.76 \mathrm{~m}$ high and is also fixed in the same rectangular boxed bars at its ends. This platform is welded up with individual plates with different thickness (see details in Fig.10). A computational model has been prepared in accordance with the geometrical dimensions listed above and using the following finite elements: 2022 beam two-noded linear elements, 20 truss two-noded linear elements and 4120 4-noded quadrilateral thin and isotropic shell elements joined in 6022 nodes (FEM discretization is shown in Fig.11). 


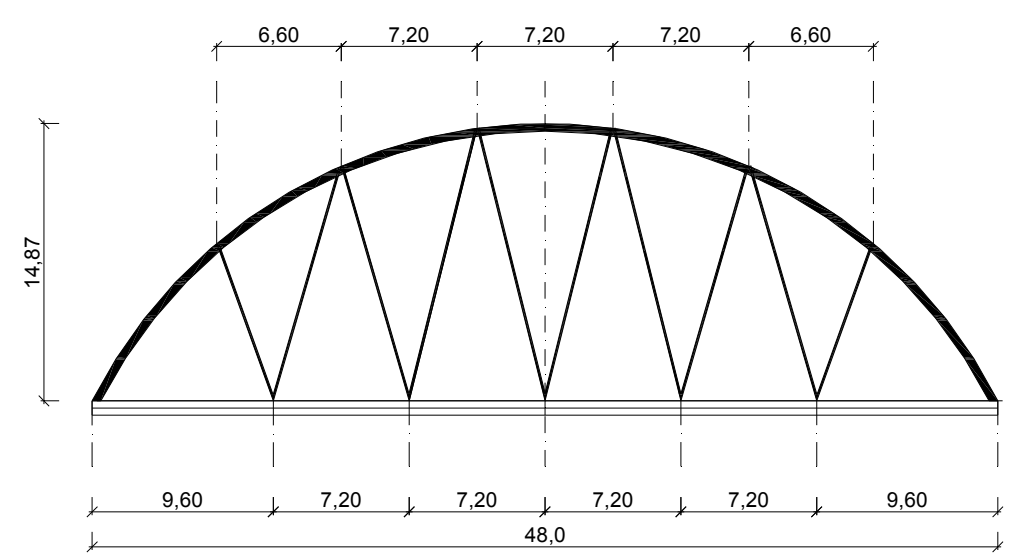

Fig.9. Basic dimensions of the analyzed bridge for pedestrians.

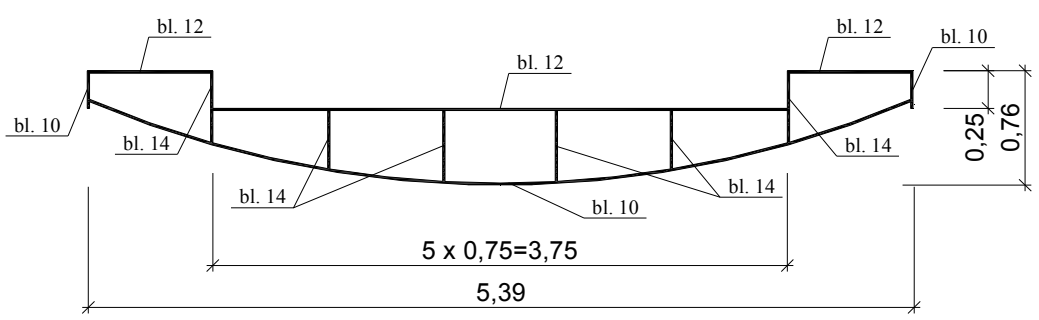

Fig.10. Cross - section of the platform.

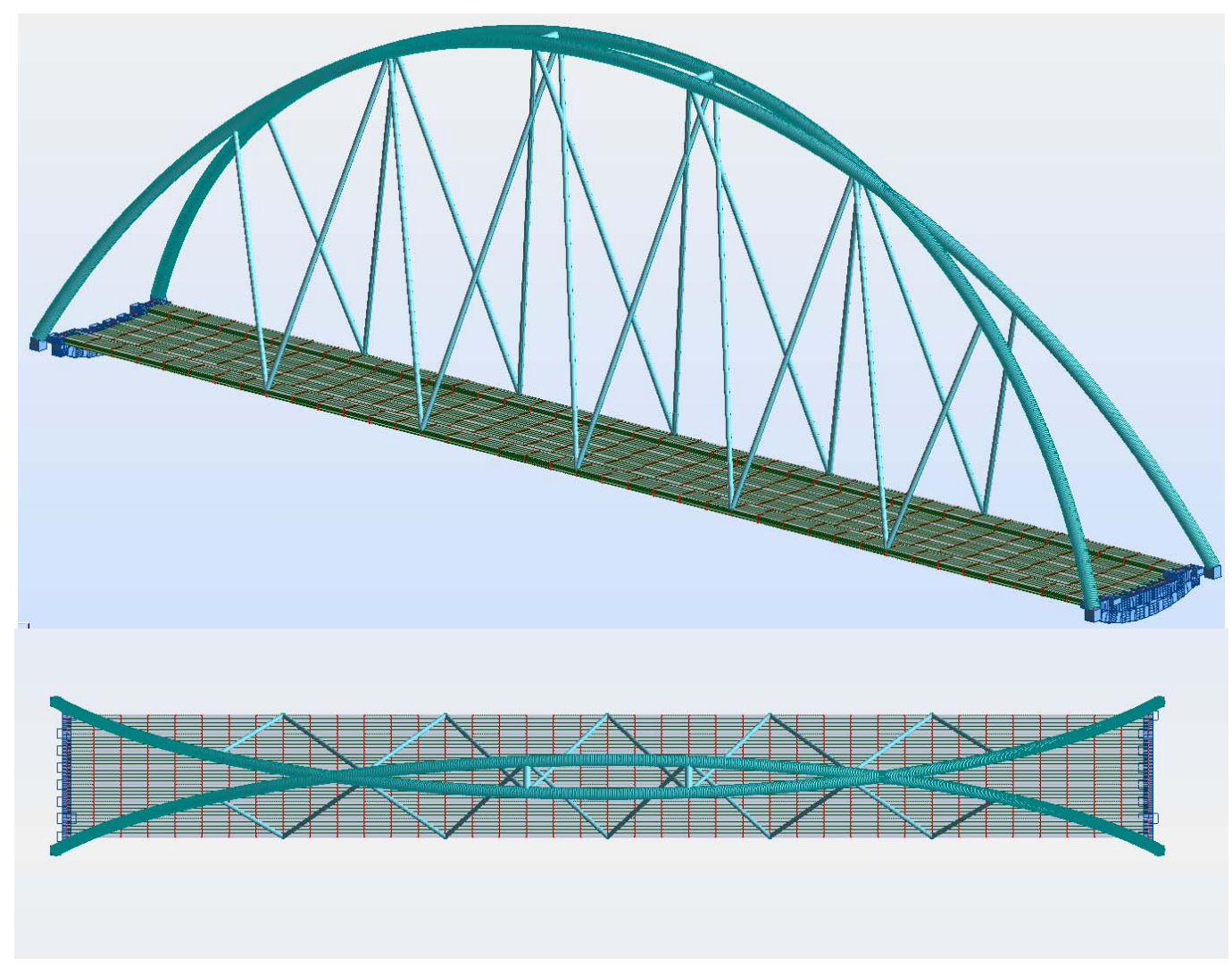

Fig.11. Computational model of the footbridge, general view. 
Four types of stochastic computations have been carried out with the use of a computational model described above: (a) analysis of the eigenfrequencies of the bridge with respect to the random thickness of plates in the box platform, (b) analysis of the eigenfrequencies of the bridge with respect to the random Young modulus of steel plates in the box platform, (c) analysis of displacements of the platform with respect to the random value of static pedestrian crowd load, (d) analysis of displacements of the platform with respect to the random thickness of plates in the box platform. All random parameters given above are treated here as uncorrelated Gaussian random variables (each analysis contains a single random parameter only).
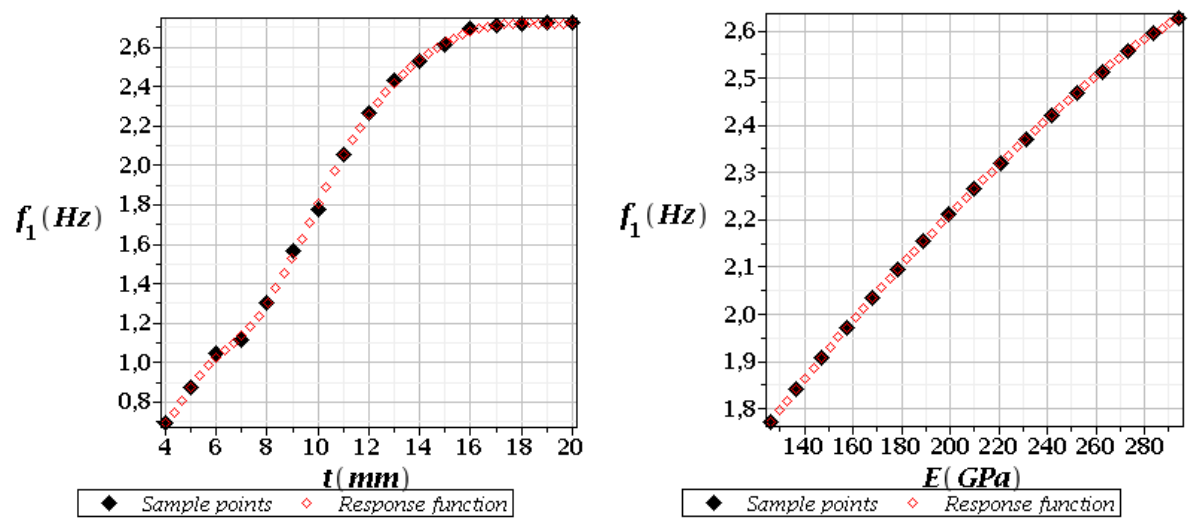

Fig.12. Response functions for eigenvibration analysis with respect to the random thickness of platform plates (left) and random Young modulus (right).

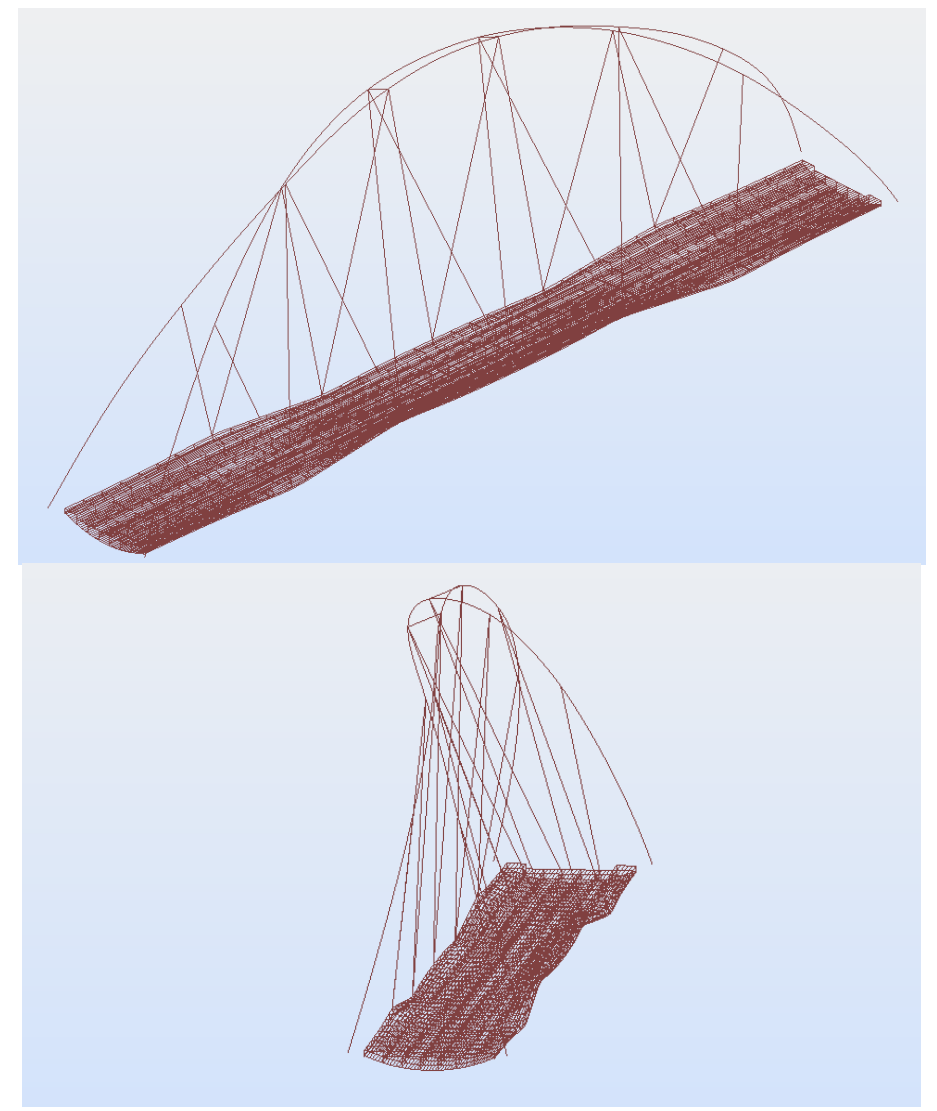

Fig.13. Deformed shape for the first mode of eigenvibrations. 

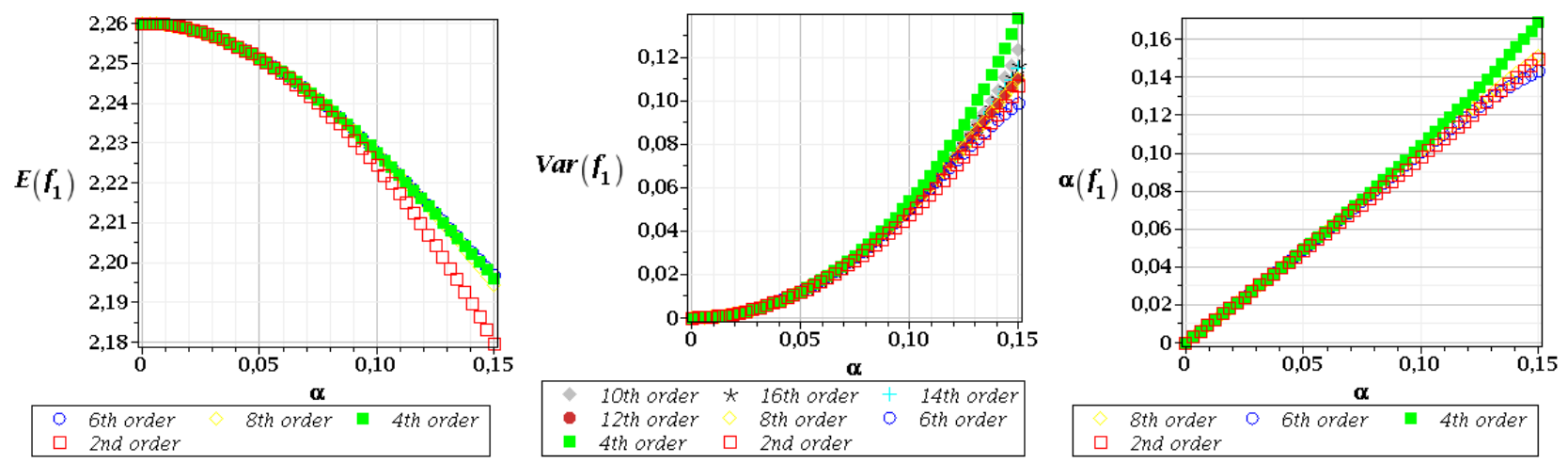

Fig.14. Expected value (left) variance (middle) and coefficient of variation for the $1^{\text {st }}$ eigenfrequency random thickness of platform plates.
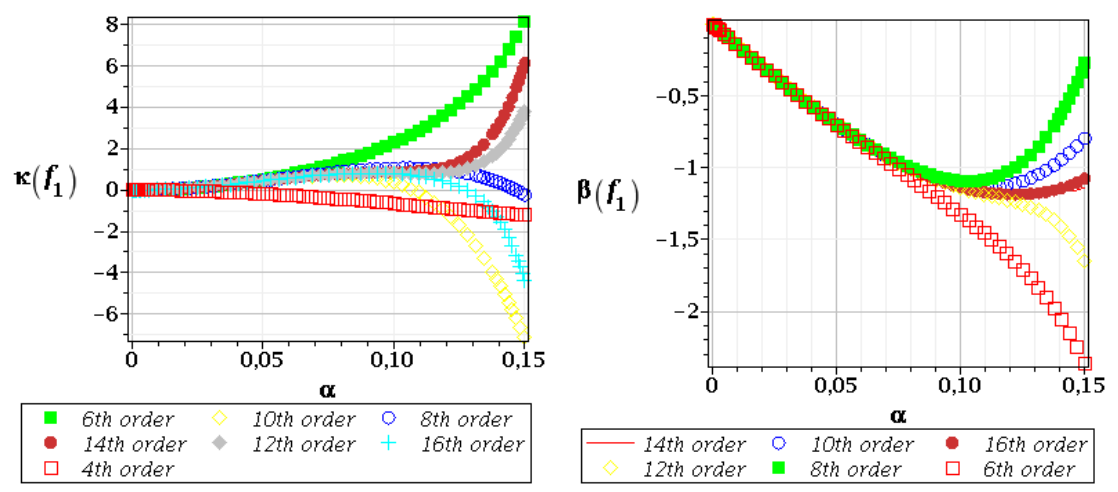

Fig.15. Kurtosis (left) and skewness (right) for the $1^{\text {st }}$ eigenfrequency - random thickness of platform plates.
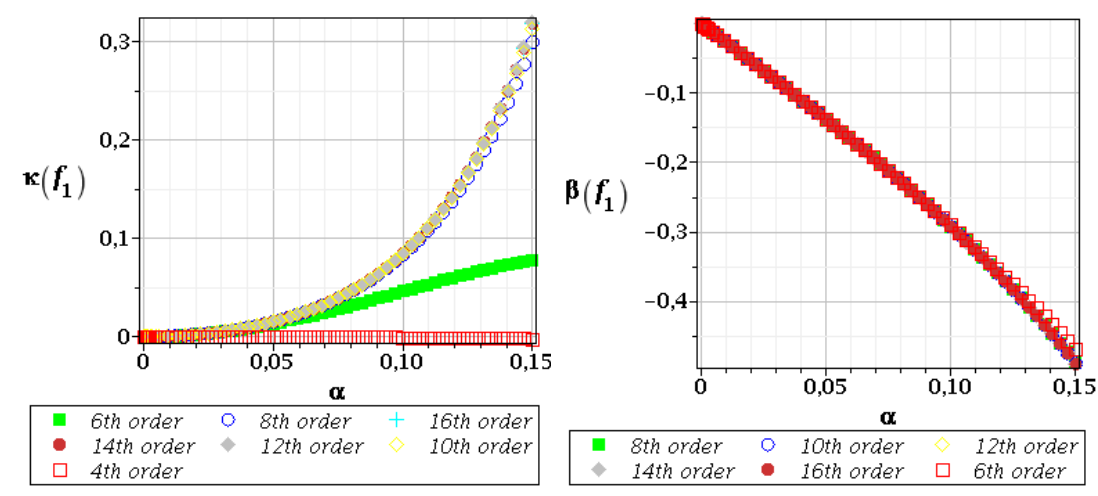

Fig.16. Kurtosis (left) and skewness (right) for the $1^{\text {st }}$ eigenfrequency - random Young modulus.

We have presented first the response function curves for bridge eigenfrequency with respect to two random parameters - thickness of plates in the box platform (left) and Young modulus of structural steel of the same platform, see Fig.12. As we can notice both functions reflect with good accuracy the numerical analysis that has been carried out (marked on the graphs as a black solid diamond sign - sample points) and they passed through all these points without any local oscillations, irregularities and singularities (especially on the both ends of a range). A function in the first numerical example has a more complex character - it 
shows also that this kind of uncertainty is much more critical for the eigenvibrations. A response function for the random Young modulus is almost linear what has a decisive impact on the particular probabilistic moments of all orders and also on an overall convergence of the perturbation method itself. In addition to Fig. 13, the deformed shape for the first mode of bridge eigenvibrations is presented and shows a dominating twisting character. It is not surprising as both arches are disconnected and unfastened in a direction perpendicular to their planes they belong to.

Probabilistic output for the structural response is given in Fig.14 for the random thickness of the plates as the expectation (left), variance (middle) and coefficient of variation (right) - all plotted with respect to the input coefficient of variation $\alpha[0 ; 0.15]$. The expected values $E\left[f_{l}\right]$ are characterized by quite a significant dispersion what could be anticipated after earlier observation of the additional response function. The same conclusion can be drawn on the variance $\operatorname{Var}\left(f_{l}\right)$ and it must be noticed that probabilistic convergence of the perturbation method in this case is visualized for curves of higher, namely: the $14^{\text {th }}$ and $16^{\text {th }}$ order. Of course the variance increases together with simultaneously increasing the input coefficient of variation. An influence of the input dispersion on the output coefficient of variance $\alpha\left(f_{l}\right)$ shows a linear dependence but it is worth underling that satisfactory convergence is obtained for higher orders curves only.

Skewness and kurtosis of the fundamental eigenvibrations are given in Fig. 15 - all the curves have much more complex forms as both parameters diverge together with an increasing input coefficient of variation; this is the main reason to extend the stochastic perturbation analysis up to the sixteenth order. Probabilistic parameters presented above give us an opportunity to characterize the output distribution function. We can conclude that the probability density function of random eigenvibrations is asymmetrical, sharp, left sided inclined, which is very significant in the reliability analysis. It means that the final distribution is non-Gaussian, and this fact does make it possible to determine and apply the Cornel reliability index given in Eurocode.

For comparison in Fig.16, the graphs contain skewness and kurtosis of eigenvibrations, but this time computed with respect to the random Young modulus. As it was mentioned above, the response functions strongly affect final statistical characteristics, especially in the context of the generalized stochastic perturbation method. Convergence represented by the curves corresponding to the different perturbation approaches in this case is much higher than that given for the random thickness of plates, especially for skewnees. We can also say that the output probability density function for eigenvibrations with respect to the random Young modulus is normal as both higher orders coefficients (skewness and kurtosis) take very small values, almost equal to 0 . It is justified also by the form of the stiffness matrix - depending linearly upon the elastic modulus within the entire structure.
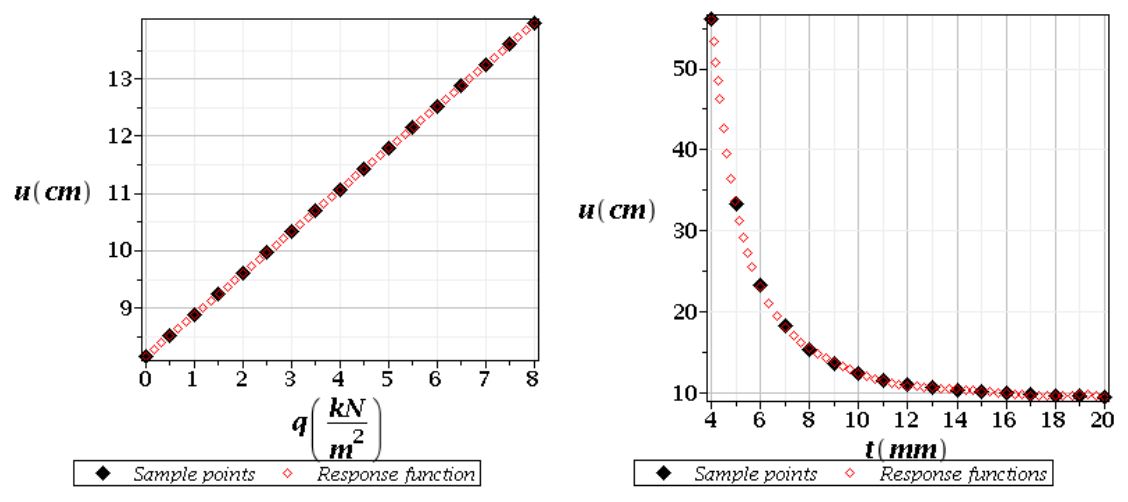

Fig.17. Response functions for maximum vertical displacements analysis with respect to random crowd of pedestrian load (left) and random thickness of platform plates (right). 


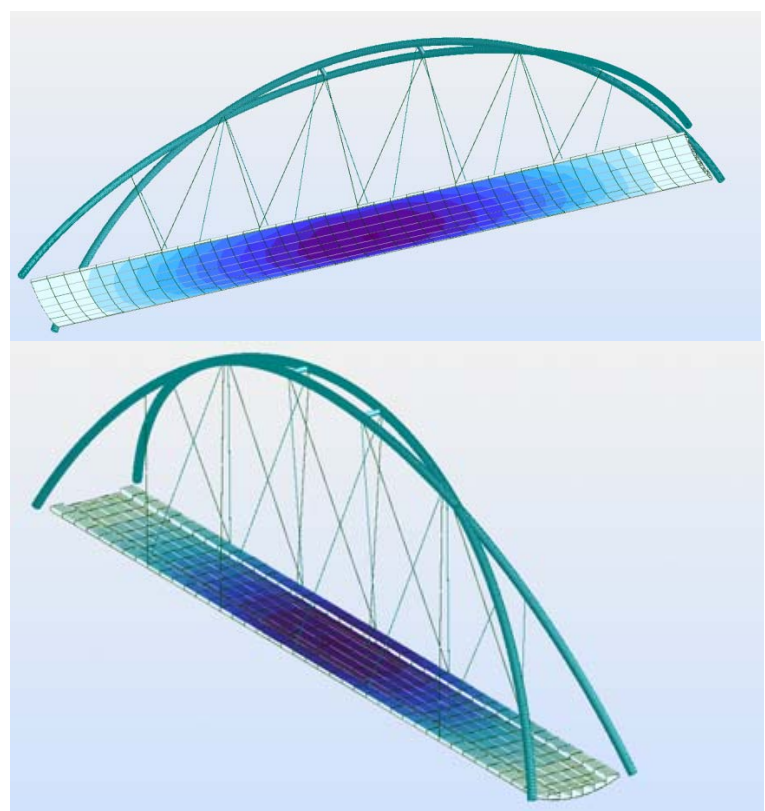

Fig.18. Vertical displacements for bottom and top of the platform under pedestrian crowd.

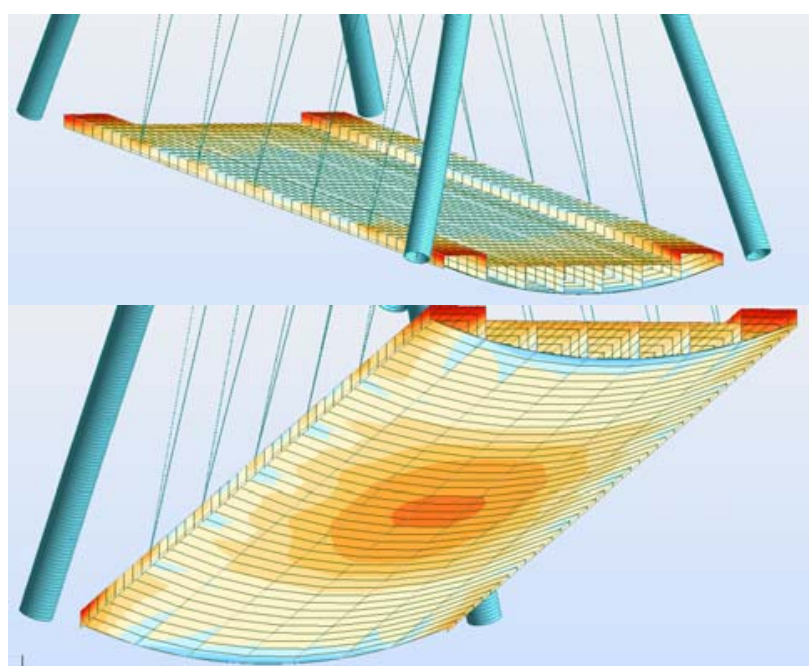

Fig.19. Stresses distributions in the platform under pedestrian crowd.
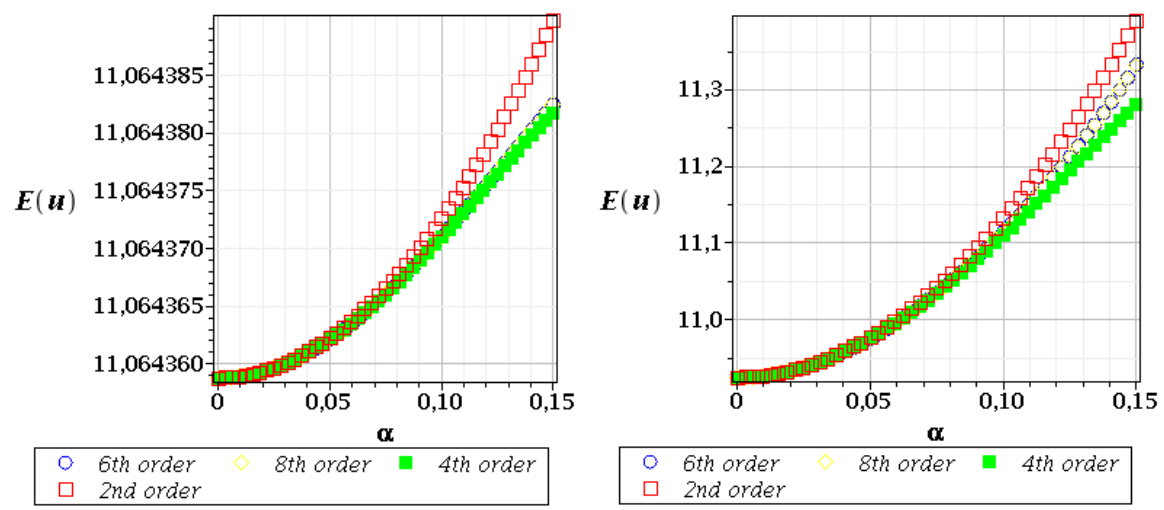

Fig.20. Expected values of maximal vertical displacement for random crowd of pedestrian load (left) and random thickness of platform plates (right). 

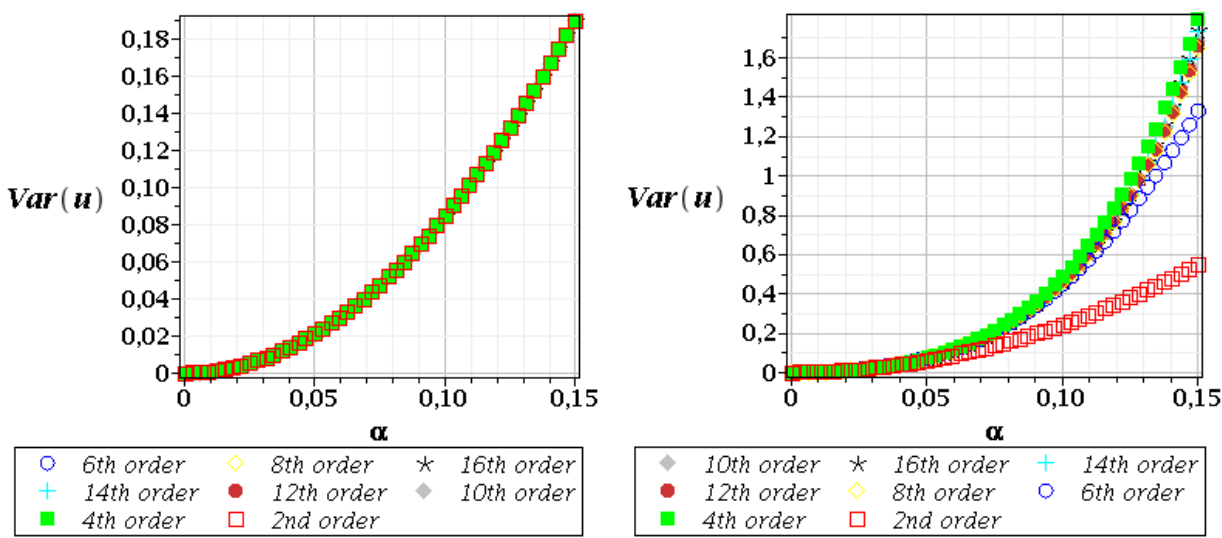

Fig.21. Variances of maximum vertical displacement for random pedestrian crowd (left) and random thickness of platform plates (right).
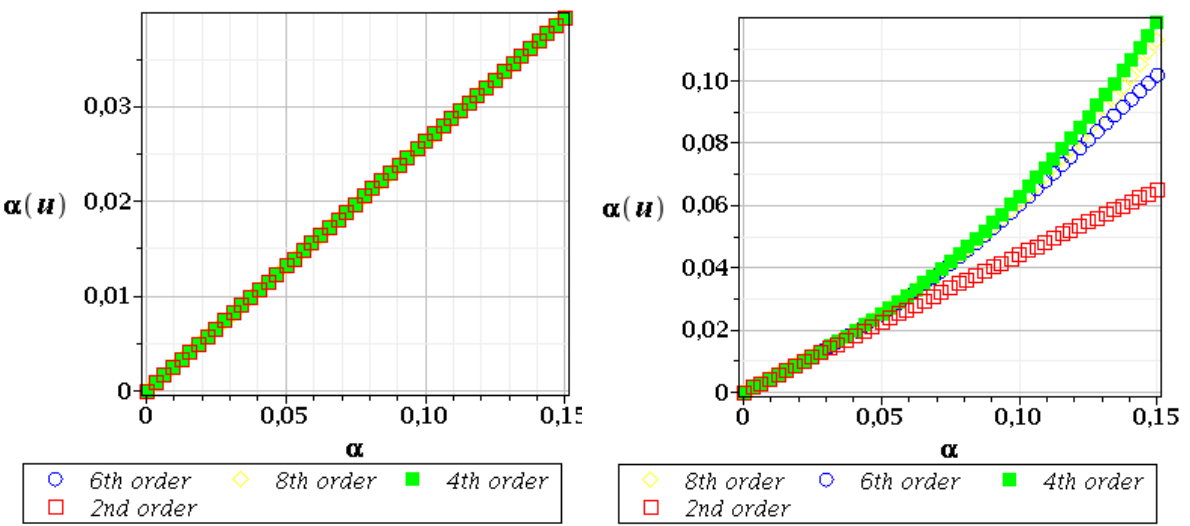

Fig.22. Coefficients of variation of maximal vertical displacement for random pedestrian crowd (left) and random thickness of platform plates (right).
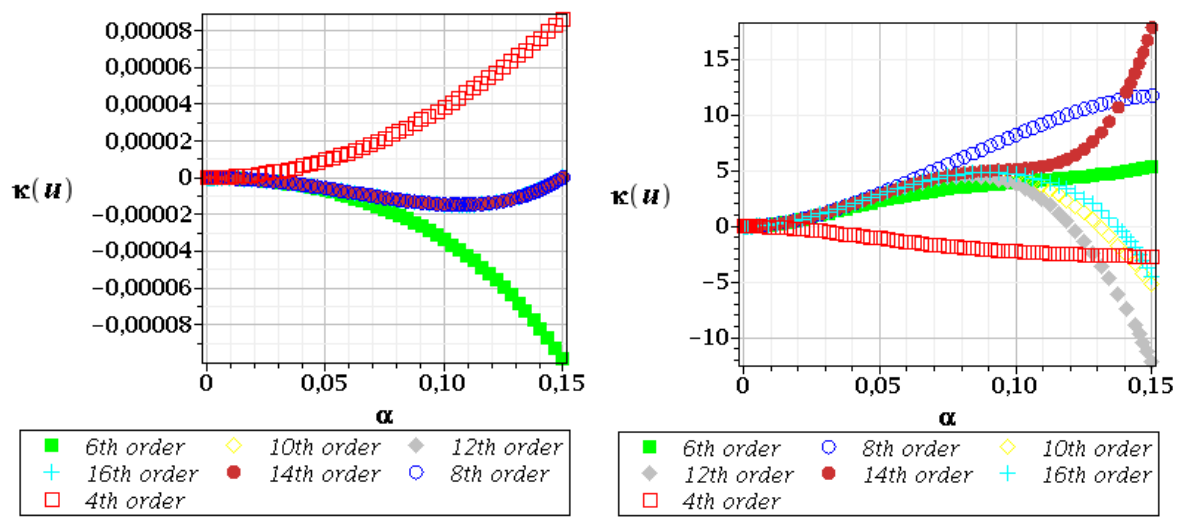

Fig.23. Kurtosis of maximum vertical displacement for random pedestrian crowd (left) and random thickness of platform plates (right). 

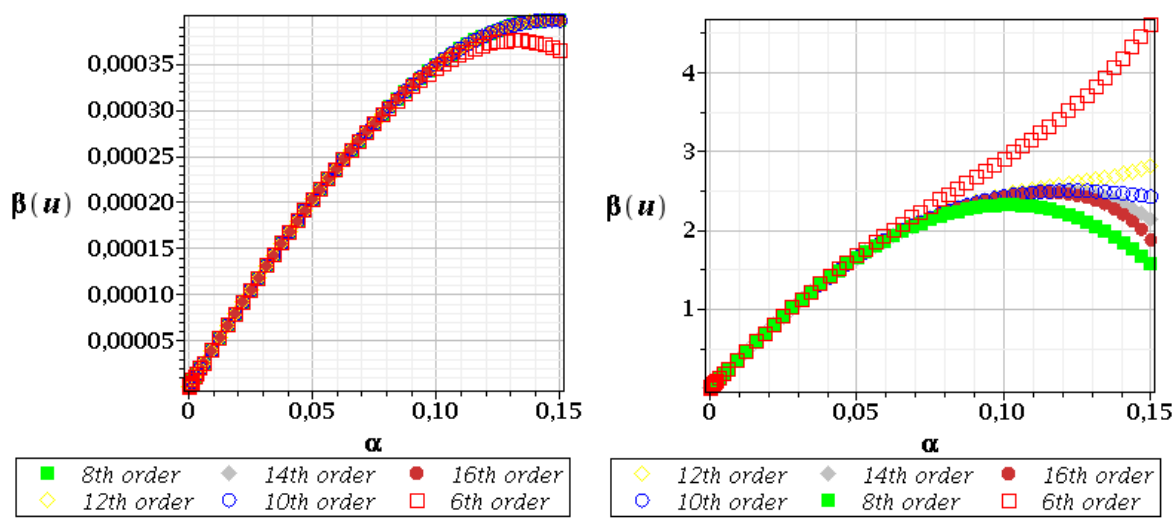

Fig.24. Skewness of maximum vertical displacement for random pedestrian crowd (left) and random thickness of platform plates (right).

The conclusions formulated above may find their consequences also in the next two stochastic analysis. As it is listed above in this section, we present the results concerning vertical displacements of the bridge platform with respect to two random parameters - static pedestrian crowd and separately, the thickness of the plates. We have response functions for these analyze (random $q$-left, random $u$-right) in Figs $17-17$ computational experiments have been performed to obtain them (marked with black solid diamonds). As can be seen, the dependence of displacement $u$ to $q$ is absolutely linear, contrary to the thickness of plates $t$, where beyond some critical value of plates thickness we notice a rapid growth of displacement values.

The maps of vertical displacements of the platform under pedestrian crowd and stresses in the same load case are given in Figs 18 and 19, respectively.

Expectations of the maximum displacements $E[u]$ given in Fig.20 increase together with increasing of the coefficient of input random dispersion and also show that an expansion of the eighth order appears to be efficient enough, even for the case of random thickness.

An analysis of variance of the displacements $u$ (Fig.21) once again shows differences resulting from the response functions $-\operatorname{Var}(u)$ for random static load (left graph) gives us the same results for each curve which shows that an expansion of the $4^{\text {th }}$ order is good enough for this specific case. Quite different results we have for the random thickness of the plates (right graph) - we have obtained numerical convergence for the curves related to the perturbation approach of order $14^{\text {th }}$ and $16^{\text {th }}$. What is natural in both cases, the values of the variance increase with an additional increase of the input parameter $\alpha$.

The output coefficient of variation $\alpha(u)$ presented in Fig.22 as a function of input $\alpha$ for a case with the random load of pedestrian crowd (left graph) does not reflect the linear dependence between these two characteristics. An increase of the input coefficient of variation up to $15 \%$ yields $4 \%$ increase in output $\alpha$ only. Higher values of $\alpha(u)$ obtained in random thickness analysis (Fig.22, right graph) show that this particular random variable has a meaningful influence on the displacement observations.

We have presented in Figs 23 and 24 kurtosis and skewness for both computational cases - random static load (left graphs) and random thickness (right graphs). The observations of these two probabilistic parameters lead us to the following conclusions: (a) both skewness and kurtosis have really small values, almost equal to 0 , which corresponds to the normal distribution, (b) the output probability density function is very similar to Gaussian distribution, (c) we can conclude that stochastic Taylor expansion of the $8^{\text {th }}$ order is quite sufficient for this specific computational experiment.

A numerical analysis with random thickness of the plates in a platform box section is much more complex than for uncertainty in the elastic modulus. Final values of kurtosis for the input coefficient of variation $\alpha$ equal to 0.10 for higher order perturbation expansions give the results around the value 5, which is characteristic for very oblate probability distribution functions. Is worth underling that for $\alpha=0.15$ we have not obtained the same results of kurtosis for curves describing approaches of $12^{\text {th }}, 14^{\text {th }}$ and $16^{\text {th }}$ order. It leads 
us to the conclusion that for complex computations and large input random dispersion there is a need to apply larger probabilistic expansions to get satisfactory probabilistic convergence.

\section{Conclusions}

1. The computational analysis performed shows that the Generalized Stochastic Finite Element Method may be used efficiently to determine first four probabilistic moments and characteristics of the structural response of the steel pedestrian bridges. An application of the perturbation - based on SFEM is unique here as we applied a combination of various finite elements (elastic bars, beams and shells) into a single numerical model. This approach enables verification of important information in the designing process. The resulting eigenfrequencies are non-Gaussian variables while randomizing Young modulus and thickness of the main girders. An analogous verification for the structural displacements shows that Gaussian character is preserved for an uncertainty in the elastic modulus only. It practically means that a simple reliability index contained in Eurocode 0 based on the fundamental Cornell model may be useless in most of practical engineering calculations.

2. Perturbation analysis of the tenth order is usually quite sufficient to accurately determine the first two probabilistic moments but determination of the next two moments demands higher order expansions. We have proposed the $18^{\text {th }}$ order perturbation approach to determine efficiently the $3^{\text {rd }}$ order moments (and also skewness) as well as the $20^{\text {th }}$ order approach to efficiently compute the fourth order probabilistic characteristics (together with kurtosis) with sufficient accuracy. It is possible thanks to the common usage of the generalized SFEM and the Response Function Method realized with the Least Square Method and polynomial basis, where any partial derivative with respect to the random input is derived analytically.

\section{Nomenclature}

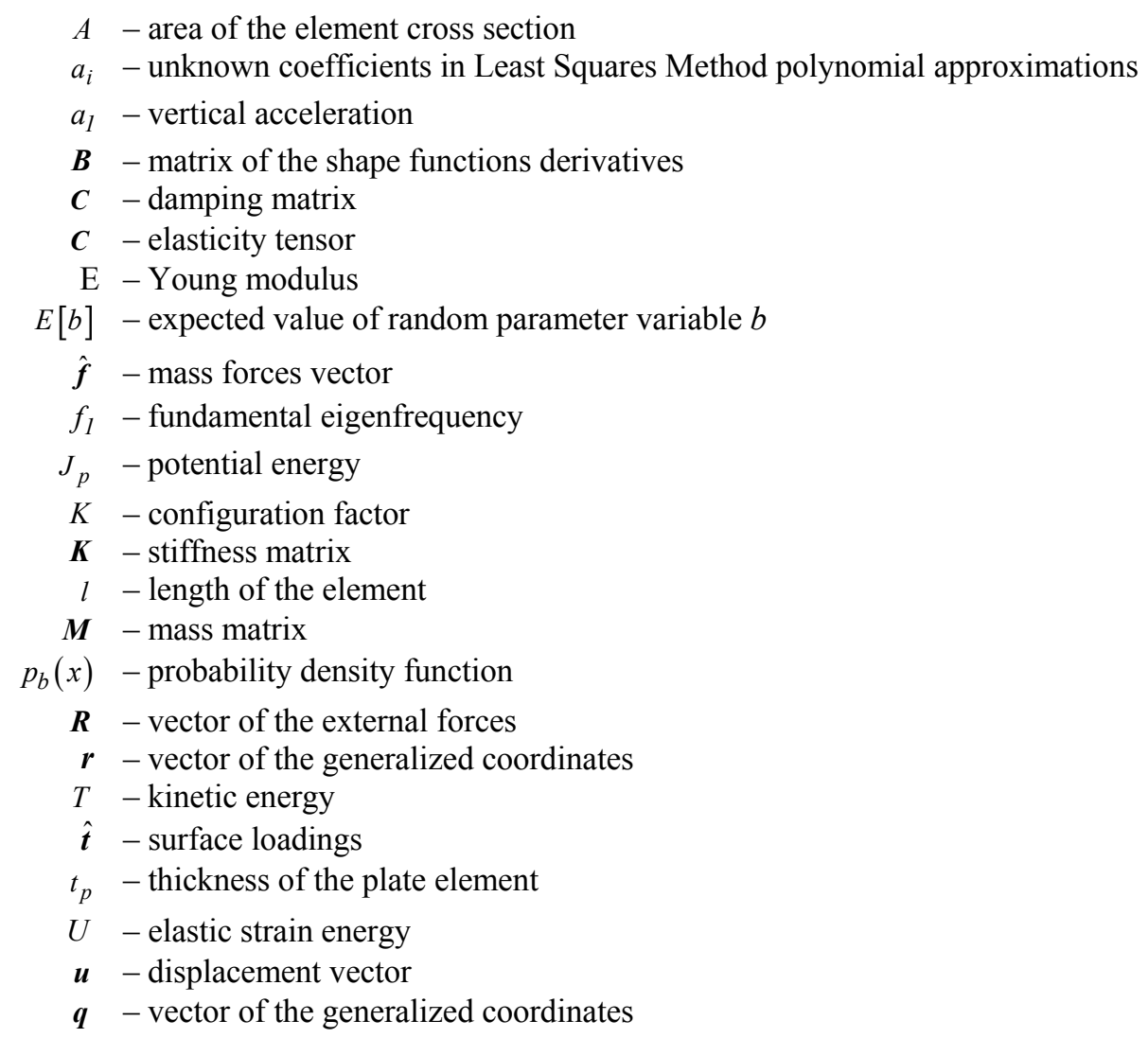




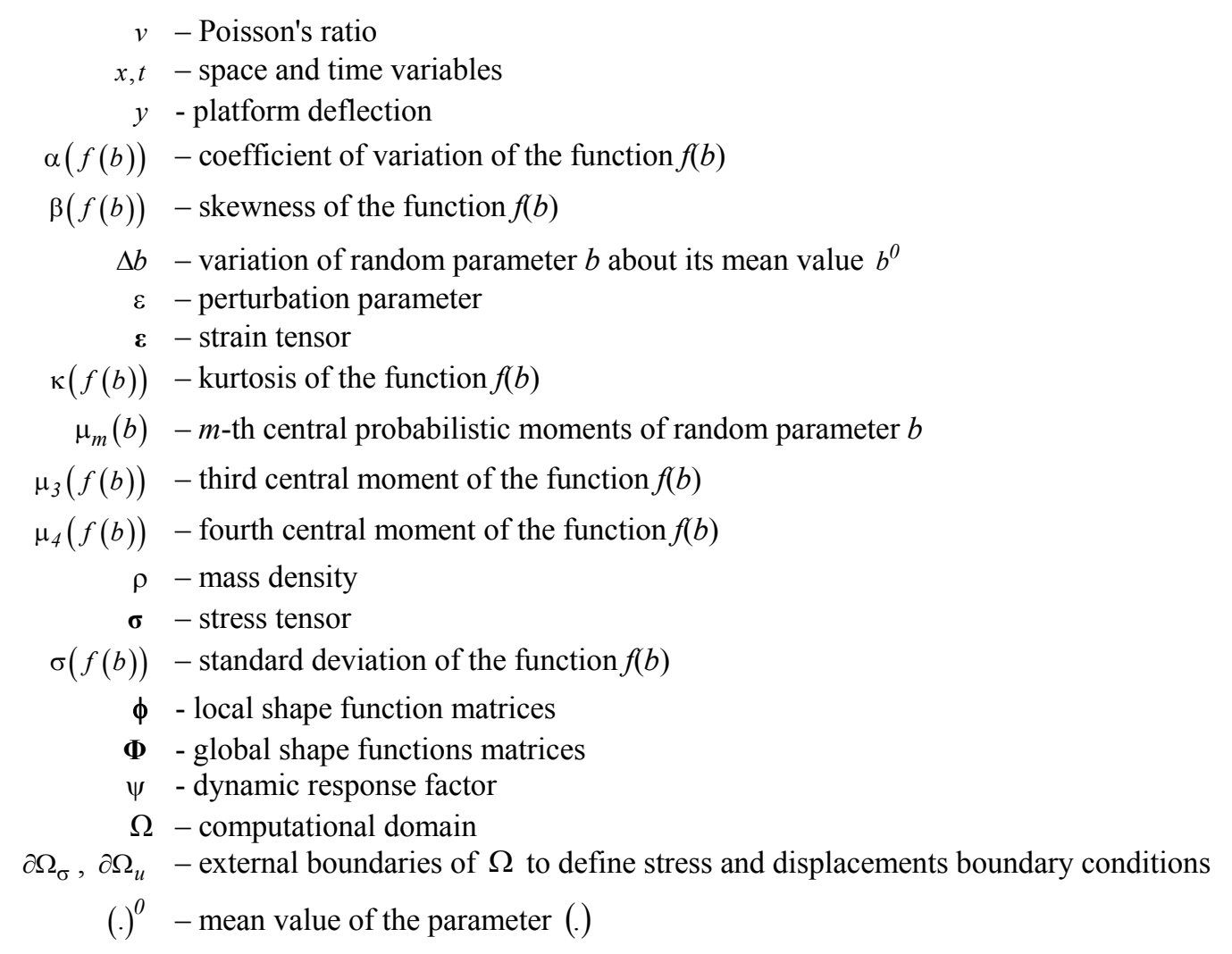

\section{References}

[1] Biliszczuk J., Barcik W. and Machelski Cz. (2007): Design of steel footbridges (in Polish). - Wrocław: Dolnośląskie Wydawnictwo Edukacyjne.

[2] Flaga A. (2011): Bridges for pedestrians (in Polish). - Warsaw: Wydawnictwo Komunikacji i Łączności.

[3] Charles P. and Hoorpah W. (2006): Technical guide - Footbridges - Assessment of vibrational behaviour of footbridges under pedestrian loading. - Paris: Setra/AFGC.

[4] Fujino Y. (2002): Vibration, control and monitoring of long-span bridges. Recent research, developments and practice in Japan. - Journal of Constructional Steel Research, vol.58, pp.71-97.

[5] Fujino Y., Pacheco B.M., Nakamura S.I. and Warnitchai P. (1993): Synchronization of human walking observed during lateral vibration of a congested pedestrian bridge. - Earthquake Engineering and Structural Dynamics, vol.22, pp.741-758.

[6] Newland D.E. (1993): Radom Vibrations and Spectral Analysis. - Harlow: Longman Group.

[7] Bathe K.J. (1996): Finite Element Procedures. Englewood Cliffs. - New York: Prentice Hall.

[8] Hughes T.J.R. (2000): The Finite Element Method - Linear Static and Dynamic Finite Element Analysis. - New York: Dover Publications, Inc.

[9] Zienkiewicz O.C. and Taylor R.L. (2005): Finite Element Method for Solid and Structural Mechanics. $6^{\text {th }}$ edition. - Amsterdam: Elsevier.

[10] Clough R.W. and Penzien J. (1975): Dynamics of Structures. - New York: McGraw-Hill.

[11] Kamiński M. (2005): Computational Mechanics of Composite Materials. Sensitivity, Randomness and Multiscale Behaviour. - London-New York: Springer-Verlag.

[12] Kleiber M. and Hien T.D. (1992): The Stochastic Finite Element Method. - Chichester: Wiley. 
[13] Bendat J.S. and Piersol A.G. (1971): Random Data: Analysis and Measurement Procedures. - New York: Wiley.

[14] Feller W. (1965): An Introduction to Probability Theory and its Applications. - New York: Wiley.

[15] Pradlwater H.J., Schueller G.I. and Szekely G.S. (2002): Random eigenvalue problems for large systems. Computers and Structures, vol.80, No.27-30, pp.2415-2424.

[16] Kamiński M. (2013): The Stochastic Perturbation Method for Computational Mechanics. - Chichester: Wiley.

Received: May 30, 2016

Revised: October 16, 2016

\section{Appendix}

Below are the formulas describing the second, third and fourth central probabilistic moments in higher order terms of the perturbation approach.

Order

Variance

$$
\begin{aligned}
& \operatorname{Var}^{16}[f(b)]=\left[\frac{\partial f(b)}{\partial b}\right]^{2} \varepsilon^{2} \mu_{2}(b)+\left[\frac{1}{4}\left(\frac{\partial f^{2}(b)}{\partial b^{2}}\right)^{2}+\frac{1}{3}\left(\frac{\partial f(b)}{\partial b}\right)\left(\frac{\partial f^{3}(b)}{\partial b^{3}}\right)\right] \varepsilon^{4} \mu_{4}(b)+ \\
& +\left[\frac{1}{36}\left(\frac{\partial f^{3}(b)}{\partial b^{3}}\right)^{2}+\frac{1}{24}\left(\frac{\partial f^{2}(b)}{\partial b^{2}}\right)\left(\frac{\partial f^{4}(b)}{\partial b^{4}}\right)+\frac{1}{60}\left(\frac{\partial f(b)}{\partial b}\right)\left(\frac{\partial f^{5}(b)}{\partial b^{5}}\right)\right] \varepsilon^{6} \mu_{6}(b)+ \\
& +\left[\begin{array}{l}
\frac{1}{576}\left(\frac{\partial f^{4}(b)}{\partial b^{4}}\right)^{2}+\frac{1}{360}\left(\frac{\partial f^{3}(b)}{\partial b^{3}}\right)\left(\frac{\partial f^{5}(b)}{\partial b^{5}}\right)+\frac{1}{720}\left(\frac{\partial f^{2}(b)}{\partial b^{2}}\right)\left(\frac{\partial f^{6}(b)}{\partial b^{6}}\right)+ \\
+\frac{1}{2520}\left(\frac{\partial f(b)}{\partial b}\right)\left(\frac{\partial f^{7}(b)}{\partial b^{7}}\right)
\end{array}\right] \varepsilon^{8} \mu_{8}(b)+ \\
& +\left[\begin{array}{l}
\frac{1}{14400}\left(\frac{\partial f^{5}(b)}{\partial b^{5}}\right)^{2}+\frac{1}{8640}\left(\frac{\partial f^{4}(b)}{\partial b^{4}}\right)\left(\frac{\partial f^{6}(b)}{\partial b^{6}}\right)+\frac{1}{15120}\left(\frac{\partial f^{3}(b)}{\partial b^{3}}\right)\left(\frac{\partial f^{7}(b)}{\partial b^{7}}\right)+ \\
+\frac{1}{40320}\left(\frac{\partial f^{2}(b)}{\partial b^{2}}\right)\left(\frac{\partial f^{8}(b)}{\partial b^{8}}\right)
\end{array}\right] \varepsilon^{10} \mu_{10}(b)+ \\
& +\left[\frac{1}{514800}\left(\frac{\partial f^{6}(b)}{\partial b^{6}}\right)^{2}+\frac{1}{302400}\left(\frac{\partial f^{5}(b)}{\partial b^{5}}\right)\left(\frac{\partial f^{7}(b)}{\partial b^{7}}\right)+\frac{1}{483840}\left(\frac{\partial f^{4}(b)}{\partial b^{4}}\right)\left(\frac{\partial f^{8}(b)}{\partial b^{8}}\right)\right] \varepsilon^{12} \mu_{12}(b)+ \\
& +\left[\frac{1}{25401600}\left(\frac{\partial f^{7}(b)}{\partial b^{7}}\right)^{2}+\frac{1}{14515200}\left(\frac{\partial f^{6}(b)}{\partial b^{6}}\right)\left(\frac{\partial f^{8}(b)}{\partial b^{8}}\right)\right] \varepsilon^{14} \mu_{14}(b)+ \\
& +\frac{1}{1625702400}\left(\frac{\partial f^{8}(b)}{\partial b^{8}}\right)^{2} \varepsilon^{16} \mu_{16}(b) \text {. }
\end{aligned}
$$


Order

Third central moment

$$
\begin{aligned}
& \mu_{3}{ }^{18}[f(b)]=\frac{3}{2}\left(\frac{\partial f(b)}{\partial b}\right)^{2}\left(\frac{\partial f^{2}(b)}{\partial b^{2}}\right) \varepsilon^{4} \mu_{4}(b)+ \\
& +\left[\frac{1}{8}\left(\frac{\partial f^{2}(b)}{\partial b^{2}}\right)^{3}+\frac{1}{2}\left(\frac{\partial f(b)}{\partial b}\right)\left(\frac{\partial f^{2}(b)}{\partial b^{2}}\right)\left(\frac{\partial f^{3}(b)}{\partial b^{3}}\right)+\frac{1}{8}\left(\frac{\partial f(b)}{\partial b}\right)^{2}\left(\frac{\partial f^{4}(b)}{\partial b^{4}}\right)\right] \varepsilon^{6} \mu_{6}(b)+ \\
& {\left[\frac{1}{24}\left(\frac{\partial f^{2}(b)}{\partial b^{2}}\right)\left(\frac{\partial f^{3}(b)}{\partial b^{3}}\right)^{2}+\frac{1}{32}\left(\frac{\partial f^{2}(b)}{\partial b^{2}}\right)^{2}\left(\frac{\partial f^{4}(b)}{\partial b^{4}}\right)+\right.} \\
& ++\frac{1}{24}\left(\frac{\partial f(b)}{\partial b}\right)\left(\frac{\partial f^{3}(b)}{\partial b^{3}}\right)\left(\frac{\partial f^{4}(b)}{\partial b^{4}}\right)+\frac{1}{40}\left(\frac{\partial f(b)}{\partial b}\right)\left(\frac{\partial f^{2}(b)}{\partial b^{2}}\right)\left(\frac{\partial f^{5}(b)}{\partial b^{5}}\right)+\varepsilon^{8} \mu_{8}(b)+ \\
& +\frac{1}{240}\left(\frac{\partial f(b)}{\partial b}\right)^{2}\left(\frac{\partial f^{6}(b)}{\partial b^{6}}\right) \\
& {\left[\frac{1}{384}\left(\frac{\partial f^{2}(b)}{\partial b^{2}}\right)\left(\frac{\partial f^{4}(b)}{\partial b^{4}}\right)^{2}+\frac{1}{288}\left(\frac{\partial f^{3}(b)}{\partial b^{3}}\right)^{2}\left(\frac{\partial f^{4}(b)}{\partial b^{4}}\right)+\right.} \\
& ++\frac{1}{480}\left(\frac{\partial f(b)}{\partial b}\right)\left(\frac{\partial f^{4}(b)}{\partial b^{4}}\right)\left(\frac{\partial f^{5}(b)}{\partial b^{5}}\right)+\frac{1}{240}\left(\frac{\partial f^{2}(b)}{\partial b^{2}}\right)\left(\frac{\partial f^{3}(b)}{\partial b^{3}}\right)\left(\frac{\partial f^{5}(b)}{\partial b^{5}}\right)+\varepsilon^{10} \mu_{10}(b)+ \\
& +\frac{1}{960}\left(\frac{\partial f^{2}(b)}{\partial b^{2}}\right)^{2}\left(\frac{\partial f^{6}(b)}{\partial b^{6}}\right)+\frac{1}{720}\left(\frac{\partial f(b)}{\partial b}\right)\left(\frac{\partial f^{3}(b)}{\partial b^{3}}\right)\left(\frac{\partial f^{6}(b)}{\partial b^{6}}\right) \\
& {\left[\frac{1}{13824}\left(\frac{\partial f^{4}(b)}{\partial b^{4}}\right)^{3}+\frac{1}{2880}\left(\frac{\partial f^{3}(b)}{\partial b^{3}}\right)\left(\frac{\partial f^{4}(b)}{\partial b^{4}}\right)\left(\frac{\partial f^{5}(b)}{\partial b^{5}}\right)+\right.} \\
& ++\frac{1}{9600}\left(\frac{\partial f^{2}(b)}{\partial b^{2}}\right)\left(\frac{\partial f^{5}(b)}{\partial b^{5}}\right)^{2}+\frac{1}{8640}\left(\frac{\partial f^{3}(b)}{\partial b^{3}}\right)^{2}\left(\frac{\partial f^{6}(b)}{\partial b^{6}}\right)+ \\
& {\left[+\frac{1}{5760}\left(\frac{\partial f^{2}(b)}{\partial b^{2}}\right)\left(\frac{\partial f^{4}(b)}{\partial b^{4}}\right)\left(\frac{\partial f^{6}(b)}{\partial b^{6}}\right)+\frac{1}{14400}\left(\frac{\partial f(b)}{\partial b}\right)\left(\frac{\partial f^{5}(b)}{\partial b^{5}}\right)\left(\frac{\partial f^{6}(b)}{\partial b^{6}}\right)\right]} \\
& +\left[\begin{array}{l}
\frac{1}{115200}\left(\frac{\partial f^{4}(b)}{\partial b^{4}}\right)\left(\frac{\partial f^{5}(b)}{\partial b^{5}}\right)^{2}+\frac{1}{86400}\left(\frac{\partial f^{3}(b)}{\partial b^{3}}\right)\left(\frac{\partial f^{5}(b)}{\partial b^{5}}\right)\left(\frac{\partial f^{6}(b)}{\partial b^{6}}\right)+ \\
+\frac{1}{138240}\left(\frac{\partial f^{4}(b)}{\partial b^{4}}\right)^{2}\left(\frac{\partial f^{6}(b)}{\partial b^{6}}\right)+\frac{1}{345600}\left(\frac{\partial f^{2}(b)}{\partial b^{2}}\right)\left(\frac{\partial f^{6}(b)}{\partial b^{6}}\right)^{2}
\end{array}\right] \varepsilon^{14} \mu_{14}(b)+ \\
& +\left[\frac{1}{3456000}\left(\frac{\partial f^{5}(b)}{\partial b^{5}}\right)^{2}\left(\frac{\partial f^{6}(b)}{\partial b^{6}}\right)+\frac{1}{4147200}\left(\frac{\partial f^{4}(b)}{\partial b^{4}}\right)\left(\frac{\partial f^{6}(b)}{\partial b^{6}}\right)^{2}\right] \varepsilon^{16} \mu_{16}(b)+ \\
& +\frac{1}{373248000}\left(\frac{\partial f^{6}(b)}{\partial b^{6}}\right)^{3} \varepsilon^{18} \mu_{18}(b) \text {, }
\end{aligned}
$$




\section{Order}

$20^{\text {th }}$

\section{Fourth central moment}

$$
\begin{aligned}
& \mu_{4}{ }^{20}[f(b)]=\left(\frac{\partial f(b)}{\partial b}\right)^{4} \varepsilon^{4} \mu_{4}(b)+\left[\frac{3}{2}\left(\frac{\partial f^{2}(b)}{\partial b^{2}}\right)^{2}\left(\frac{\partial f(b)}{\partial b}\right)^{2}+\frac{2}{3}\left(\frac{\partial f(b)}{\partial b}\right)^{3}\left(\frac{\partial f^{3}(b)}{\partial b^{3}}\right)\right] \varepsilon^{6} \mu_{6}(b)+ \\
& +\left[\begin{array}{l}
\frac{1}{16}\left(\frac{\partial f^{2}(b)}{\partial b^{2}}\right)^{4}+\frac{1}{2}\left(\frac{\partial f(b)}{\partial b}\right)\left(\frac{\partial f^{2}(b)}{\partial b^{2}}\right)^{2}\left(\frac{\partial f^{3}(b)}{\partial b^{3}}\right)+\frac{1}{6}\left(\frac{\partial f(b)}{\partial b}\right)^{2}\left(\frac{\partial f^{3}(b)}{\partial b^{3}}\right)^{2}+ \\
+\frac{1}{4}\left(\frac{\partial f(b)}{\partial b}\right)^{2}\left(\frac{\partial f^{2}(b)}{\partial b^{2}}\right)\left(\frac{\partial f^{4}(b)}{\partial b^{4}}\right)+\frac{1}{30}\left(\frac{\partial f(b)}{\partial b}\right)^{3}\left(\frac{\partial f^{5}(b)}{\partial b^{5}}\right)
\end{array}\right] \varepsilon^{8} \mu_{8}(b)+ \\
& +\left[\begin{array}{l}
\frac{1}{54}\left(\frac{\partial f(b)}{\partial b}\right)\left(\frac{\partial f^{3}(b)}{\partial b^{3}}\right)^{3}+\frac{1}{24}\left(\frac{\partial f^{2}(b)}{\partial b^{2}}\right)^{2}\left(\frac{\partial f^{3}(b)}{\partial b^{3}}\right)^{2}+\frac{1}{48}\left(\frac{\partial f^{2}(b)}{\partial b^{2}}\right)^{3}\left(\frac{\partial f^{4}(b)}{\partial b^{4}}\right)+ \\
+\frac{1}{12}\left(\frac{\partial f(b)}{\partial b}\right)\left(\frac{\partial f^{2}(b)}{\partial b^{2}}\right)\left(\frac{\partial f^{3}(b)}{\partial b^{3}}\right)\left(\frac{\partial f^{4}(b)}{\partial b^{4}}\right)+\frac{1}{96}\left(\frac{\partial f(b)}{\partial b}\right)^{2}\left(\frac{\partial f^{4}(b)}{\partial b^{4}}\right)^{2}+ \\
+\frac{1}{40}\left(\frac{\partial f(b)}{\partial b}\right)\left(\frac{\partial f^{2}(b)}{\partial b^{2}}\right)^{2}\left(\frac{\partial f^{5}(b)}{\partial b^{5}}\right)+\frac{1}{60}\left(\frac{\partial f(b)}{\partial b}\right)^{2}\left(\frac{\partial f^{3}(b)}{\partial b^{3}}\right)\left(\frac{\partial f^{5}(b)}{\partial b^{5}}\right)^{2}
\end{array}\right] \varepsilon^{10} \mu_{10}(b)+ \\
& +
\end{aligned}
$$

